\title{
Charged domain walls as quantum strings on a lattice
}

\author{
Henk Eskes, ${ }^{*}$ Osman Yousif Osman, Rob Grimberg, Wim van Saarloos, and Jan Zaanen \\ Instituut-Lorentz, Leiden University, P.O. Box 9506, NL-2300 RA Leiden, The Netherlands
}

(Received 30 December 1997; revised manuscript received 8 May 1998)

\begin{abstract}
Recently, experimental evidence has been accumulating that the doped holes in the high- $T_{c}$ cuprate superconductors form domain walls separating antiferromagnetic domains. These so-called stripes are linelike objects and if these persist in the superconducting state, high- $T_{c}$ superconductivity is related to a quantum string liquid. In this paper the problem of a single quantum meandering string on a lattice is considered. A kink model is introduced for the string dynamics, which allows us to analyze lattice commensuration aspects. Building on earlier work by den Nijs and Rommelse [Phys. Rev. B 40, 4709 (1989)], this lattice string model can be related both to restricted solid-on-solid models, describing the world-sheet of the string in Euclidean space time, and to one-dimensional quantum spin chains. At zero temperature a strong tendency towards orientational order is found and the remaining directed string problem can be treated in detail. Quantum delocalized strings are found whose long-wavelength wandering fluctuation is described by free field theory and it is argued that the fact that the critical phase of delocalized lattice strings corresponds to a free Gaussian theory is a very general consequence of the presence of a lattice. In addition, the mapping on the surface problem is exploited to show the existence of different types of localized string phases; some of these are characterized by a proliferation of kinks, but the kink flavors are condensed so that the long-wavelength fluctuations of these strings are suppressed. The simplest phase of this kind is equivalent to the incompressible (Haldane) phase of the $S=1$ spin chain and corresponds to a bond centered string: The average string position is centered on bonds. We also find localized phases of this type that take arbitrary orientations relative to the underlying lattice. The possible relevance of these lattice strings for the stripes in cuprates is discussed. [S0163-1829(98)05132-7]
\end{abstract}

\section{INTRODUCTION}

A series of experimental developments has changed the perspective on the problem of high- $T_{c}$ superconductivity drastically. As long as the doping level is not too high, electrons bind at temperatures well above $T_{c}$ (Ref. 1) and the superconducting state appears to be in tight competition with some collective insulating state. ${ }^{2}$ There exists compelling evidence that this insulating state corresponds with a different type of electron crystal, characterized by both spin and charge condensation: the stripe phase. ${ }^{3-5}$ This phase consists of a regular array of charged magnetic domain walls: The holes introduced by doping form linelike textures that are at the same time antiphase boundaries, separating antiferromagnetic spin domains; see Fig. 1(a). This stripe phase is observed in systems where the insulating state is stabilized by $\mathrm{Zn}$ doping ${ }^{5,6}$ or by the so-called low temperature tetragonal (LTT) collective pinning potential. ${ }^{3,4}$

Inelastic neutron scattering data reveal that strong dynamical stripe correlations persist in the metallic and superconducting regimes. ${ }^{4,6-8}$ Although no static stripe order is present, the magnetic fluctuations as measured by inelastic neutron scattering should reflect stripe correlations. As was shown very recently, the magnetic modulation wave vector of the static stripe phase seems identical to that of the dynamical spin fluctuations in the metal and superconductor for various doping levels. ${ }^{4}$ In addition, it was argued that the anomalous normal state magnetic dynamics can be explained in terms of domain wall meandering dynamics. ${ }^{9}$

The exciting possibility arises that the zero-temperature superconducting state is at the same time a relatively mildly fluctuating quantum stripe fluid. Unlike the rather featureless diagonal sector of, e.g., ${ }^{4} \mathrm{He},{ }^{10}$ it can be imagined that the charge and spin sectors of this quantum stripe problem have an interesting internal structure. Because charged domain walls are linelike objects, the charge sector might be looked at as a quantum string liquid. ${ }^{9,11,12}$ Little is known in general about such problems and a theoretical analysis is needed. In order to address the problem of many interacting strings, it is first necessary to find out the physics of a single string or charged domain wall in isolation. A string is an extended (a)

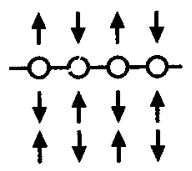

(c)

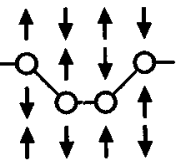

(e)

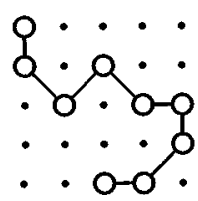

(b)

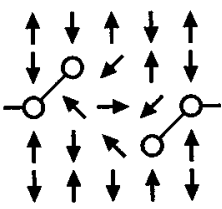

(d)

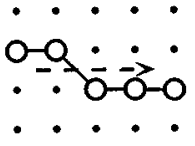

(f)

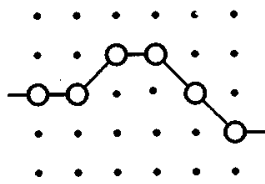

FIG. 1. (a) Charged domain wall separating spin domains of opposite antiferromagnetic order parameter. (b) Breaking up domain walls causes spin frustration, while (c) "kinks" do not. (d) Kinks can gain kinetic energy by moving along the domain wall. (e) Typical rough wall. (f) Example of a directed string. 
object, carrying a nontrivial collective dynamics: In contrast to particlelike problems, the elementary constituent of the string liquid poses already a serious problem. The physics of quantum strings is a rich subject. This is most easily discussed in terms of path integrals. In $(D+1)$-dimensional Euclidean space time, a particle corresponds to a world line and so the quantum string corresponds to a "world sheet.", The statistical physics of membranes is a rich subject, which is still under active investigation. ${ }^{13}$

The debate on the microscopic origin of the stripe instability is far from closed. ${ }^{12,14-19}$ Nevertheless, in this paper we will attempt to isolate some characteristics that might be common to all present proposals for the microscopy to arrive at some general considerations regarding the quantum meandering dynamics. From those we will abstract a minimal model for the string dynamics. The phase diagram of this model can be mapped out completely and turns out to be remarkably rich.

These characteristic features are the following. (i) It is assumed that the charge carriers are confined to domain walls. This is the major limitation of the present work and it is hoped that at least some general characteristics of this strong-coupling regime survive in the likely less strongly coupled regime where nature appears to be. (ii) In addition, we assume that domain walls are not broken up, as sketched in Fig. 1(b), as this would lead to strong spin frustration. (iii) Most importantly, we assume a dominant role of lattice commensuration on the scale of the lattice constant. Configuration space is built from strings that consist of "holes" on the sites of an underlying lattice. An example of such a string configuration is sketched in Fig. 1(c). This automatically implies that the microscopic dynamics is that of kinks along the strings [Figs. 1(c) and 1(d)] and this leads to major simplifications with regard to the long-wavelength behavior of the string as a whole. Note that there is ample evidence for the importance of lattice commensuration: the scaling of the incommensurability with hole density $x$ for $x<\frac{1}{8},{ }^{6}$ the special instability at $x=\frac{1}{8},{ }^{4}$ and the LTT pinning mechanism. ${ }^{3}$ (iv) It is assumed that the strings do not carry other low-lying internal degrees of freedom, apart from the shape fluctuations. Physically this means that localized strings would be electronic insulators. The data of Yamada et al. ${ }^{5}$ indicate that this might well be the case at dopings $x \leqslant \frac{1}{8}$ (the linear dependence of the incommensurability on $x$ indicates an ondomain wall charge commensuration), but it is definitely violated at larger dopings where the strings should be metallic. ${ }^{19-22}$ Work is in progress on fluctuating metallic strings, where we find indications that the collective string dynamics is quite similar to what is presented here for insulating strings. ${ }^{23}$

Given these requirements, one would like to consider a quantum sine-Gordon model ${ }^{24}$ for the string dynamics,

$$
H=\frac{1}{2} \int d l\left[\Pi(l)^{2}+c^{2}\left(\frac{\delta z(l)}{\delta l}\right)^{2}+g \sin \left(\frac{2 \pi z(l)}{a}\right)\right] .
$$

Here $z(l)$ is the transversal displacement at point $l$ on the string, $\Pi(l)$ is its conjugate momentum defined through the commutation relation $\left[\Pi(l), z\left(l^{\prime}\right)\right]=i \delta\left(l-l^{\prime}\right)$, and $c$ is the transversal sound velocity. The first two terms in Eq. (1.1) describe a free string, while the last term is responsible for the lattice commensuration effects: Every time the string is displaced by a lattice constant, the potential energy is at a minimum. This model is well understood. ${ }^{24}$ When the strength of the nonlinear interaction exceeds a critical value $\left(g>g_{c}\right)$, the interaction term is relevant and the string localizes. The excitation spectrum develops a gap and it is characterized by well-defined kink and antikink excitations. When $\left(g<g_{c}\right)$ the sine term is irrelevant and although the dynamics is at least initially kinklike on microscopic scales, the string behaves as a free string at long wavelength. The latter is the most elementary of all quantum strings. It follows immediately that the relative transversal displacement of two points separated by an arclength $l$ along the string diverges as $\left\langle[z(l)-z(0)]^{2}\right\rangle \sim \ln l .^{9}$ The string as a whole is therefore delocalized and this is the simplest example of a "critical', string.

A central result of this paper is that Eq. (1.1) is, at least in principle, not fully representative for the present lattice problem. More precisely, starting from a more complete microscopic kink dynamics model (Sec. II), we find a richer infrared fixed-point structure. The phase diagram incorporates phases associated with the quantum sine-Gordon model fixed point, but also includes additional phases that are intimately connected with the effects of the lattice and of the nearestneighbor interactions between the holes. In Sec. III we derive the path-integral representation of our model. It turns out that the world sheet of this string in Euclidean space time corresponds with two coupled restricted solid-on-solid (RSOS) surfaces, ${ }^{25}$ each of which describes the motion of the string in either the $x$ or $y$ direction on the two-dimensional lattice.

The bare model is invariant under rotations of the string in space. As discussed in Sec. IV, we find indications for a generic zero-temperature spontaneous symmetry breaking: For physical choices of parameters, the invariance under symmetry operations of the lattice is broken. Even when the string is critical (delocalized in space), it acquires a sense of direction. On average, the trajectories corresponding to the string configurations move always, forward in one direction, while the string might delocalize in the other direction; see Fig. 1(f). This involves an order-out-of-disorder phenomenon, which is relatively easy to understand intuitively. Quantum mechanics effectively enhances the fluctuation dimension by stretching out the string into a world sheet in the timewise direction and the enhancement of the effective dimension reduces the effect of fluctuations. Thermal fluctuations destroy this directedness, but they do so more effectively when the string is less quantum mechanical.

This directedness simplifies the remaining problem considerably. We will show that the directed string problem is equivalent to a well-known problem in surface statistical physics: Its world sheet is equivalent to a single RSOS surface. At the same time, this model is easily shown to be equivalent to a generalized $X X Z$ quantum spin-chain problem. The particular model we study is actually equivalent to the $S=1$ spin chain, which has been studied in great detail. The RSOS surface problem and the quantum spin-chain problem are therefore also related to each other. This equivalence was actually at the center of the seminal work of den Nijs and Rommelse on the hidden order in Haldane spin chains. ${ }^{26}$ From our perspective, the introduction of the physi- 
cally appealing quantum string model as an intermediate model that connects both with the spin chain and the RSOS surfaces also helps to appreciate the depth of the work of den Nijs and Rommelse. ${ }^{26}$

The bulk of this paper (Secs. V-VIII) is devoted to an exhaustive treatment of this directed string model. Some powerful statistical physics notions apply directly to the present model and these allow us to arrive at a complete description of the phase diagram of the quantum string. As was mentioned already in Ref. 11, this phase diagram is surprisingly rich: There are in total ten distinct phases. In the context of the quantum spin-chain/RSOS surfaces, already six of those were previously identified. However, viewing this problem from the perspective of the quantum string, it becomes natural to consider a larger number of potentially relevant operators and the other four phases become obvious.

Compared to strings described by Eq. (1.1), we find a much richer behavior, but this is limited to the regime where lattice commensuration dominates over the kinetic energy so that the string as a whole is localized. We use "localized" here in the sense that the transversal string fluctuations of two widely separated points remain finite, $\left\langle[z(l)-z(0)]^{2}\right\rangle$ $\rightarrow$ const as $l \rightarrow \infty$. Besides the different directions the purely classical strings can take in the lattice, we also find a number of localized strings that have a highly nontrivial internal structure: the "disordered flat" strings, characterized by a proliferation of kinks, but where the kink flavors condense so that the string as a whole remains localized. On the other hand, the quantum-delocalized (critical) strings are all of the free-field variety and as we will argue in Sec. IX, this might be a very general consequence of the presence of a lattice cutoff.

\section{MODEL: THE MEANDERING LATTICE STRING}

Whatever one thinks about the microscopy of the stripes, in the end any theory will end up considering the charged domain walls as a collection of particles bound to form a connected trajectory, or such a model will be an important ingredient of it. Moreover, these trajectories will communicate with the crystal lattice because the electrons from which the strings are built do so as well. This fact alone puts some strong constraints on the collective dynamics of the charged domain walls.

Let us consider the string configuration space. On the lattice this will appear as a collection of particles on lattice sites, while every particle is connected to two other particles via links connecting pairs of sites. The precise microscopic identity of these particles is unimportant: They might be single holes (filled charged domain walls ${ }^{14,15}$ as in the nickelates ${ }^{27}$ ), an electron-hole pair (the charge-density waves of Nayak and Wilczek ${ }^{12}$ or Zaanen and Olés ${ }^{18}$ ), or a piece of metallic $^{20}$ or even superconducting ${ }^{28}$ domain wall. All that matters is that these entities have a preferred position with regard to the underlying lattice (site ordered ${ }^{14}$ or bond ordered $^{19}$ ). Quite generally, curvature will cost potential energy and a classical string will therefore be straight, oriented along one of the high-symmetry directions of the lattice. Without loss of generality, it can be assumed that the lattice is a square lattice while the string lies along the $(1,0)(x)$ direction. Denoting as $N_{y}$ the number of lattice sites in the $y$ direction and assuming periodic boundary conditions, this straight string can be positioned in $N_{y}$ ways on the lattice. Obviously, such a string will delocalize by local quantum moves: The particles tunnel from site to site. ${ }^{17,29}$ Moving the whole string one position in the $y$ direction involves an infinity of local moves in the thermodynamic limit and the different classical strings occupy dynamically disconnected regions of Hilbert space.

This is analogous to what is found in one-dimensional systems with a discrete order parameter. ${ }^{30}$ In the case of, e.g., polyacetylene the order parameter is of the $Z_{2}$ kind: The bond order wave can be either $\cdots-A-B-A-B-\cdots$ or $\cdots-B-A-B-A-\cdots$ ( $A$ is a single bond and $B$ a double bond), while a single translation over the lattice constant transforms the first state of the staggered order parameter into the second kind of state. This is a discrete operation because the lattice forces the bond order to localize on the center of the bonds. Such an order parameter structure implies the existence of topological defects, which are Ising domain walls: $\cdots-A-B-A-B-B-A-B-A-\cdots$ ("kink") and $\cdots-B-A-B-A-A-B-A-B-\cdots$ ("antikink"). When they occur in isolated form, these are also genuine building blocks for the quantum dynamics because although their energy is finite, it involves an infinity of local moves to get rid of them (topological stability). In the particular problem of polyacytelene, these kinks only proliferate under doping (charged solitons). Although topological quantum numbers are no longer strictly obeyed when the density of topological defects is finite, it has been shown in a number of cases that they nevertheless remain genuine ultraviolet quantities as long as they do not overlap too strongly. ${ }^{31,32}$

If we consider a (locally) directed piece of string, the string is analogous, except that the symmetry is now $Z_{N_{y}}$ : On the torus, a half infinity of the string is localized at the $y$ position $n_{y}$ and the other half can be displaced to $n_{y}+1$, $n_{y}+2, \ldots, n_{y}-1$. Hence, in total there are $N_{y}-1$ distinct kink excitations with the topological invariants corresponding to the net displacement of the half string in the $y$ direction. Because the kink operators can occur in many flavors, this problem is therefore in principle richer than that of onedimensional solids.

Clearly, kinks with different flavors have to be dynamically inequivalent. Since there is apparently a reason for the particles to form connected trajectories, it should be more favorable to create a kink corresponding to a small displacement than one corresponding to a large jump. Here we will focus on the simplest possibility: Only kinks occur corresponding to a displacement of one lattice constant in the $y$ direction. This restriction is physically motivated by the fact that the string is thought to separate two antiferromagnetically ordered states; so, if the displacement of successive holes would be larger than one lattice constant, the antiferromagnetic ordering would be strongly suppressed: After all, this is the very reason that holes tend to line up in stripes. In addition, we will specialize on the "neutral" string. It will be assumed that the string is characterized by a gap in its charge and spin excitation spectrum, so that the strings with kinks contain the same number of particles as the classical reference configurations. The model we will consider might apply literally to the charge commensurate stripes of the nickelate. ${ }^{27}$ In the cuprates, it might be better to consider the 
(a)

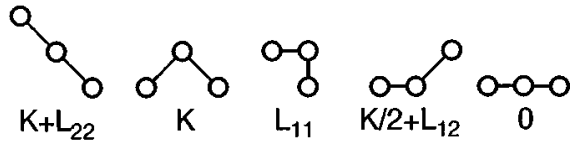

(b)

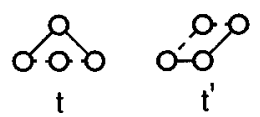

FIG. 2. (a) Set of local configurations and their classical energies. (b) Two allowed hopping processes. We take $t=t^{\prime}$.

stripes as one-dimensional metals or superconductors, characterized by massless internal excitations. In these cases, it remains to be demonstrated that eventually the transversal string fluctuations decouple from the internal excitations for the present model to be of relevance.

Given these considerations, we propose the following model for quantum lattice strings. The string configurations are completely specified by the positions of the particles (holes) $\mathbf{r}_{l}=\left(x_{l}, y_{l}\right)$ on the two-dimensional (2D) square lattice. Two successive particles $l$ and $l+1$ can only be nearest or next-nearest neighbors, or $\left|\mathbf{r}_{l+1}-\mathbf{r}_{l}\right|=1$ or $\sqrt{2}$. We will call these connections between successive particles links. Two classes of links, those of length 1 and those of length $\sqrt{2}$, exist. Taking the order of the particles into account, there are eight distinct links. The string Hilbert space is spanned by all real-space configurations satisfying the above string constraint.

We consider local discretized string-tension interactions between nearest and next-nearest holes in the chain $(\mathcal{H}$ $=-\beta H)$

$$
\begin{aligned}
\mathcal{H}_{C l}= & \sum_{l}\left[\mathcal{K} \delta\left(\left|x_{l+1}-x_{l}\right|-1\right) \delta\left(\left|y_{l+1}-y_{l}\right|-1\right)\right. \\
& \left.+\sum_{i, j=0}^{2} \mathcal{L}_{i j} \delta\left(\left|x_{l+1}-x_{l-1}\right|-i\right) \delta\left(\left|y_{l+1}-y_{l-1}\right|-j\right)\right] \\
& +\mathcal{M} \sum_{l, m} \delta\left(\mathbf{r}_{l}-\mathbf{r}_{m}\right) .
\end{aligned}
$$

The various local configurations and interaction energies are shown in Fig. 2. The last term is an excluded-volume-type interaction: The physically relevant limit is $M \rightarrow \infty$, so that holes cannot occupy the same site. The interaction $\mathcal{K}$ distinguishes horizontal from diagonal links and $\mathcal{L}_{i j}=\mathcal{L}_{j i}$ is a set of two-link interactions, which one can think of as microscopic curvature terms. Furthermore, we exclude strings with a physically unrealistic extreme curvature by taking $\mathcal{L}_{10}$ $\rightarrow \infty$. Note also that configurations that would give a contribution $\mathcal{L}_{00}$ to the energy are automatically excluded in the limit $M \rightarrow \infty$, which we will take throughout this paper. There are five local configurations, distinguished by four parameters. Therefore, we can choose $\mathcal{L}_{20}=0$ and the string is determined by the parameters $\mathcal{K}, \mathcal{L}_{11}, \mathcal{L}_{12}$, and $\mathcal{L}_{22}$; see Fig. 2.

The second term in $\mathcal{H}$ is a quantum term that allows the particles to hop to nearest-neighbor lattice positions. However, such hopping processes should not violate the string constraint. This constraint can be enforced by means of a projection operator $P_{\text {str }}\left(\mathbf{r}_{l+1}-\mathbf{r}_{l}\right)$, which restricts the motion of hole $l$ to the space of string configurations,

$$
P_{\text {str }}(\mathbf{r})=\delta(|\mathbf{r}|-1)+\delta(|\mathbf{r}|-\sqrt{2}) .
$$

The string is quantized by introducing conjugate momenta $\pi_{l}^{\alpha},\left[r_{l}^{\alpha}, \pi_{m}^{\beta}\right]=i \delta_{l, m} \delta_{\alpha, \beta}$, where $\alpha=x$ or $y$. A term $e^{i n \pi_{l}^{x}}$ acts like a ladder operator and causes particle $l$ to hop a distance $n$ in the $x$ direction,

$$
e^{i n \pi_{l}^{x}}\left|x_{l}\right\rangle=\left|x_{l}+n\right\rangle .
$$

Therefore, the kinetic-energy term becomes

$$
\mathcal{H}_{Q}=2 \mathcal{T} \sum_{l, \alpha} P_{\mathrm{str}}^{\alpha}\left(\mathbf{r}_{l+1}-\mathbf{r}_{l}\right) P_{\mathrm{str}}^{\alpha}\left(\mathbf{r}_{l}-\mathbf{r}_{l-1}\right) \cos \left(\pi_{l}^{\alpha}\right) .
$$

Note that the particles, even under $\mathcal{H}_{Q}$, keep their order and therefore can be labeled by $l$. Thus the fermion nature of holes in realistic domain walls plays no role at our level of approximation and quantum statistics becomes irrelevant.

The above model is minimal since it contains only nearest-neighbor hopping and the simplest string tension terms. One natural extension would be to take the two hopping amplitudes $\mathcal{T}$ in Fig. 2(b) to be different since there is no microscopic reason why they should be identical. In the following sections we will discuss the zero-temperature properties of the above string model. The self-avoidance term is a complicated nonlocal operator. However, we will find that, surprisingly, the kinetic energy favors oriented walls without loops. Therefore, this term turns out to be unimportant for the present zero-temperature discussion.

\section{RELATION TO RSOS-LIKE SURFACE MODELS}

The problem introduced in the preceding section can be reformulated as the classical problem of a two-dimensional surface (worldsheet) embedded in $(2+1)$-dimensional space, using the Suzuki-Trotter mapping. The model can be seen as two coupled RSOS surfaces. The solid-on-solid models are classical models for surface roughening. ${ }^{25}$ They describe stacks of atoms of integer height in two dimensions, with an interaction between adjacent stacks depending on the height differences. With this construction overhangs are excluded. In the RSOS models these height differences are limited to be smaller or equal to some integer $n$. In the present case, the two RSOS models parametrize the motion of the world sheet in the spatial $x$ and $y$ directions, respectively, while the (strong) couplings between the two takes care of the integrity of the world sheet as a whole.

In the Suzuki-Trotter ${ }^{33}$ or Feynman path-integral picture one writes the finite-temperature partition function as an infinite product over infinitesimal imaginary time slices. In this limit the commutators between the various terms in the Hamiltonian vanish like $1 / n^{2}$, where $n$ is the number of Trotter slices, and the partition function can be written as

$$
\mathcal{Z}=\lim _{n \rightarrow \infty} \operatorname{Tr}\left(e^{\mathcal{H}_{C l} / n} e^{\mathcal{H}_{Q} / n}\right)^{n} .
$$

To show the relation with RSOS models, we will cast the transfer matrices $T$ in the form of a two-dimensional classical 
effective Hamiltonian. This implies writing the matrix elements of the $T$ matrix between configurations $\left\{\mathbf{r}_{l}\right\}$ in terms of an effective classical energy depending on the world-sheet positions $\left\{\mathbf{r}_{l, k}\right\}$, where $k$ is the imaginary time index running from 1 to $n$ with periodic boundary conditions. Schematically,

$$
\lim _{n \rightarrow \infty}\left\langle\left\{\mathbf{r}_{l}\right\}_{k}\left|e^{(1 / n) \mathcal{H}}\right|\left\{\mathbf{r}_{l}\right\}_{k+1}\right\rangle \rightarrow e^{\mathcal{H}_{\mathrm{eff}}\left(\left\{\mathbf{r}_{l}\right\}_{k},\left\{\mathbf{r}_{l}\right\}_{k+1}\right)}
$$

Since $\mathcal{H}_{C l}$ is diagonal in the real-space string basis, it is already in the required form

$$
\lim _{n \rightarrow \infty}\left\langle\left\{\mathbf{r}_{l}\right\}_{k}\right| e^{(1 / n) \mathcal{H}_{C l}} \rightarrow e^{(1 / n) \mathcal{H}_{C l}\left(\left\{\mathbf{r}_{l}\right\}_{k}\right)}\left\langle\left\{\mathbf{r}_{l}\right\}_{k}\right| .
$$

For $\mathcal{H}_{Q}$ a few more steps are needed,

$$
\begin{aligned}
\left\langle\left\{\mathbf{r}_{l}\right\}_{k}\right| e^{(1 / n) \mathcal{H}_{Q}\left|\left\{\mathbf{r}_{l}\right\}_{k+1}\right\rangle} \\
=\left\langle\left\{\mathbf{r}_{l}\right\}_{k}\left|\sum_{m=0}^{\infty} \frac{1}{m !}\left(\frac{\mathcal{H}_{Q}}{n}\right)^{m}\right|\left\{\mathbf{r}_{l}\right\}_{k+1}\right\rangle \\
=\left\langle\left\{\mathbf{r}_{l}\right\}_{k}\left|1+\frac{\mathcal{H}_{Q}}{n}\right|\left\{\mathbf{r}_{l}\right\}_{k+1}\right\rangle+O\left(\frac{1}{n^{2}}\right) \\
=\prod_{l} \prod_{\alpha=x, y}\left(\delta\left(\alpha_{l, k+1}-\alpha_{l, k}\right)\right. \\
\left.+\frac{\mathcal{T}}{n} \delta\left(\left|\alpha_{l, k+1}-\alpha_{l, k}\right|-1\right)\right) \\
=\exp \left[\sum _ { l } \operatorname { l n } ( \frac { \mathcal { T } } { n } ) \left[\delta\left(\left|x_{l, k+1}-x_{l, k}\right|-1\right)\right.\right. \\
\left.+\delta\left(\left|y_{l, k+1}-y_{l, k}\right|-1\right)\right] .
\end{aligned}
$$

The expression in the last line is of course only valid for states in which the $\alpha_{l}$ 's in successive time slices differ by at most one unit. Combining these two energy contributions, we arrive at the classical problem

$$
\begin{aligned}
\mathcal{Z}=\lim _{n \rightarrow \infty} \operatorname{Tr} e^{\mathcal{H}_{\mathrm{eff}}} & \\
\mathcal{H}_{\mathrm{eff}}= & \sum_{l, k}\left[\frac{\mathcal{K}}{n} \delta\left(\left|x_{l+1, k}-x_{l, k}\right|-1\right) \delta\left(\left|y_{l+1, k}-y_{l, k}\right|-1\right)\right. \\
+ & \sum_{i, j=0}^{2} \frac{\mathcal{L}_{i j}}{n} \delta\left(\left|x_{l+1, k}-x_{l-1, k}\right|-i\right) \\
& \times \delta\left(\left|y_{l+1, k}-y_{l-1, k}\right|-j\right) \\
+ & \frac{M}{n} \sum_{m} \delta\left(x_{l, k}-x_{m, k}\right) \delta\left(y_{l, k}-y_{m, k}\right)+\ln \left(\frac{\mathcal{T}}{n}\right) \\
& \times\left[\delta\left(\left|x_{l, k+1}-x_{l, k}\right|-1\right)+\delta\left(\left|y_{l, k+1}-y_{l, k}\right|-1\right)\right]
\end{aligned}
$$

This classical world sheet is constrained to $\left|x_{l, k+1}-x_{l, k}\right|$ $\leqslant 1$ and $\left|y_{l, k+1}-y_{l, k}\right| \leqslant 1$ and the interactions are anisotropic. The above classical model can be viewed as two coupled two-dimensional RSOS surfaces $x_{l, k}$ and $y_{l, k}$. The $x$ coordinate of hole $l$ at the time slice $k$ is now identified as the height of a RSOS column positioned at $(l, k)$ in the square lattice. In a similar way the $y$ coordinates define a second RSOS surface, coupled strongly to the first by the above classical interactions. Since the steps $\Delta x$ can at most be equal to 1 , the RSOS sheets are restricted to height differences $0, \pm 1$ between neighboring columns. The classical model as defined above is not unique. While the above mapping allows us to exploit the connection to other models most efficiently, for the numerical Monte Carlo calculations a different decomposition is used, which allows for a more efficient approach to the time continuum limit. This is further discussed in Appendix A.

\section{DIRECTEDNESS AS SPONTANEOUS SYMMETRY BREAKING}

We are not aware of any similarity of the statistical physics problem of the preceding section to any existing model. RSOS problems are well understood, but it should be realized that in the present model the two RSOS problems are strongly coupled, defining a different dynamical problem. When we studied this problem with the quantum Monte Carlo method, we found a generic zero-temperature symmetry breaking: Although the string can be quantum delocalized, it picks spontaneously a direction in space. This symmetry breaking happens always in the part of parameter space that is of physical relevance.

Let us first discuss the simulations. In principle, the property of directedness is a global quantity. Consider 2D space with open boundary conditions. Directedness means that if the string starts at, say, the left boundary it will always have its end point at the right boundary and it will never reach the top or bottom boundaries. Although in our specific model it appears possible to rephrase this global property in terms of a local order parameter, a quantitative measure of directedness can be constructed that is more general. Although awkward for analytical purposes, this measure is easily evaluated numerically and it illustrates effectively the phenomenon. Every continuous string configuration $s$ can be written as a parametrized curve in two dimensions $[x(t), y(t)]$, where $t$ could, for instance, be the discrete label of the successive particles along the string. When the string configuration can be parametrized by a single-valued function $x(y)$ or $y(x)$, we call the string configuration directed [see Fig. 1(f)]. The quantum string vacuum is a linear superposition of many string configurations. When all configurations in the vacuum correspond to single-valued functions $x(y)$ or $y(x)$, the string vacuum has a directedness order parameter. At zero temperature, the ground-state wave function of the string is

$$
\left|\Psi_{0}\right\rangle=\sum_{\left\{x_{l}, y_{l}\right\}} \alpha_{0}\left(\left\{x_{l}, y_{l}\right\}\right)\left|\left\{x_{l}, y_{l}\right\}\right\rangle
$$

where every state in string configuration space $\left(\left|\left\{x_{l}, y_{l}\right\}\right\rangle\right)$ corresponds to a trajectory $[x(t), y(t)]$. Consider first the case of a continuous string. For every configuration, the total string arclength is given by

$$
L\left(\left\{x_{l}, y_{l}\right\}\right)_{\mathrm{tot}}=\int d s=\int \sqrt{d x^{2}+d y^{2}} .
$$




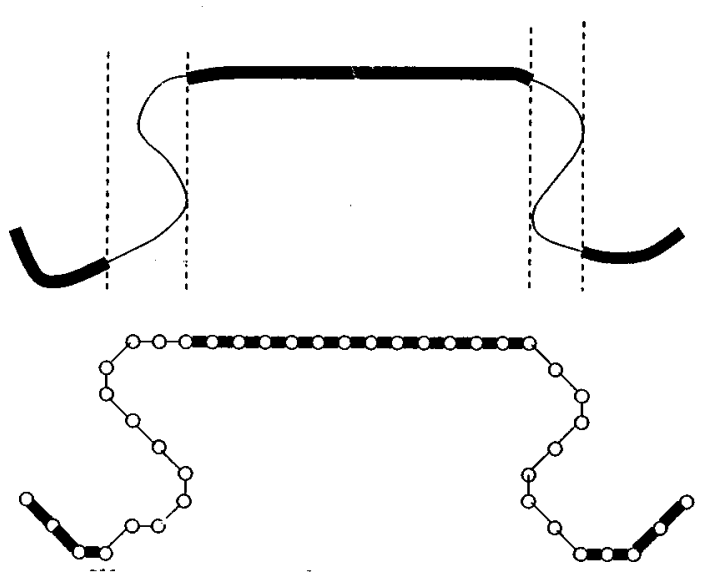

FIG. 3. Illustration of the way we measure the directedness of a string (a) in the continuum case and (b) on the lattice. The heavy solid parts of the string indicate the parts where the projection of the string onto the $x$ axis is single valued and for which the indicator function $g_{y}(x)$ equals 1 .

Consider now an indicator function $g_{y}(x)$, which equals 1 when the string is single valued when projected onto the $x$ axis and zero otherwise, and analogously a function $g_{x}(y)$, which equals 1 when the curve is single valued when projected onto the $y$ axis and zero otherwise (see Fig. 3). The total directed length in the $x$ and $y$ directions is then defined as

$$
\begin{aligned}
& L\left(\left\{x_{l}, y_{l}\right\}\right)_{\operatorname{dir}, x}=\int d x g_{y}(x) \sqrt{1+\left(\frac{d y}{d x}\right)^{2}}, \\
& L\left(\left\{x_{l}, y_{l}\right\}\right)_{\operatorname{dir}, y}=\int d y g_{x}(y) \sqrt{1+\left(\frac{d x}{d y}\right)^{2}} .
\end{aligned}
$$

The measure of directedness in the string vacuum is then defined as the larger of $N_{\mathrm{dir}}^{x}(0)$ and $N_{\mathrm{dir}}^{y}(0)$, where

$$
N_{\mathrm{dir}}^{\eta}(0)=\sum_{\left\{x_{l}, y_{l}\right\}}\left|\alpha^{0}\left(\left\{x_{l}, y_{l}\right\}\right)\right|^{2} \frac{L\left(\left\{x_{l}, y_{l}\right\}\right)_{\mathrm{dir}, \eta}}{L\left(x_{l}, y_{l}\right)_{\mathrm{tot}}}
$$

and $\eta=x, y$. On the lattice, our measure of directedness is the immediate analog of this definition, except that we just count the number of directed bonds, irrespectively of whether they are oriented diagonally or horizontally. By thermal averaging, the above definition of directedness density is immediately extended to finite temperature,

$$
N_{\mathrm{dir}}^{\eta}(T)=\sum_{n} e^{-\beta\left(E_{n}-E_{0}\right)} N_{\mathrm{dir}}^{\eta}(n),
$$

where $N_{\mathrm{dir}}^{\eta}(n)$ is the directedness density of a string excitation with energy $E_{n}$.

Equation (4.5) can be straightforwardly calculated using the quantum Monte Carlo method. A Monte Carlo snapshot defines a stack of coupled string configurations along the imaginary-time direction (the Trotter direction). We calculate $N_{\text {dir }}^{x}$ for every Trotter slice by calculating the fraction of the string length in this configuration that is single valued in the $x$ direction. This is given by the number of bonds that step forward in the $x$ direction divided by the total number of bonds in the string. We then average this quantity over the
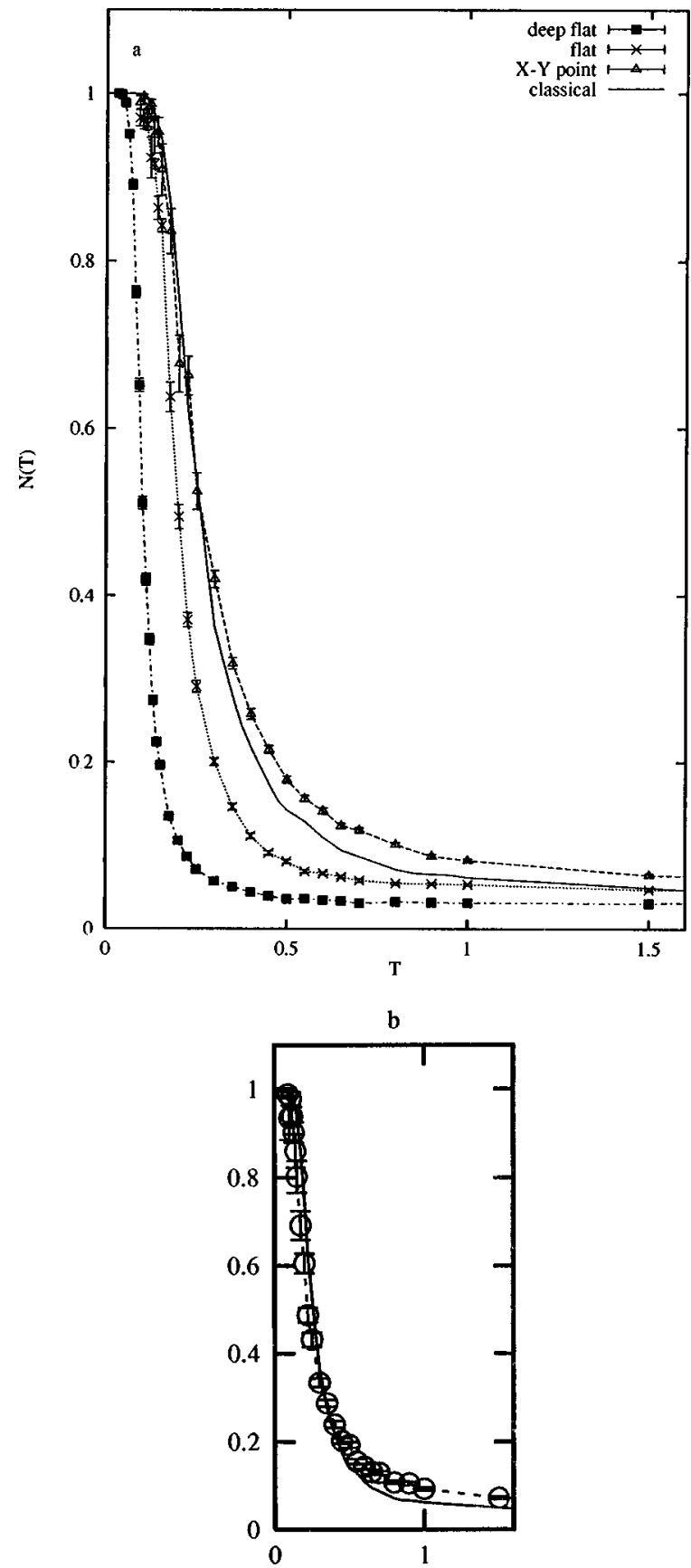

FIG. 4. Monte Carlo result for the directedness density $N_{\text {dir }}(T)$ at four points. (a) The $X Y$ point (triangles) where all curvature energies are zero. Two points are in the flat phase, with $\mathcal{K}=1.8$ (crosses) and $\mathcal{K}=4.0$ (filled squares); the rest of the curvature energies are zero. (b) Inset: a point in the middle of the Gaussian phase with parameters $\mathcal{K}=0.5, \mathcal{L}_{21}=-0.25, \mathcal{L}_{22}=-1.0$, and $\mathcal{L}_{11}$ $=0$ (open circles). The full line in both figures is the result for a classical string where only flat bonds and $\pi / 2$ corners are present with $\mathcal{L}_{11}=1$.

string world sheet (Trotter direction) and then over the Monte Carlo measurements. The same is done for $N_{\mathrm{dir}}^{y}(n)$. The larger of $N_{\mathrm{dir}}^{x}(n)$ and $N_{\mathrm{dir}}^{y}(n)$ is then the density of directedness at the given temperature.

In Fig. 4 we show the results of typical Monte Carlo calculations for the density of directedness as a function of temperature $N_{\text {dir }}(T)$. We have considered four points in the 
parameters space; as will be discussed later, these points are representative for phases with interesting quantum fluctuations and serve to clarify our conclusion. In Fig. 4(a) the triangles (dashed line) is the result for the density of directedness at the point where all the classical curvature energies are zero, i.e., corresponding to the pure quantum string. The crosses (dotted line) and the filled squares (dash-dotted line) are the results for points where $\mathcal{K}=1.8$ and 4.0 , respectively, and the rest of the classical curvature energies are zero. In terms of the phase diagram for the directed string problem of Fig. 8 in Sec. VI and Table III in Sec. VII, the first point corresponds to a Gaussian string (pure quantum) and the other two correspond to flat strings. The point $\mathcal{K}=1.8$ lies just inside the flat string phase II where significant quantum fluctuations are still present, while the point $\mathcal{K}=4.0$ lies deep inside the flat phase. The fourth curve in Fig. 4(a), given by the full line, is the result of a Monte Carlo calculation for a classical string $(\mathcal{T}=0)$ where only flat segments and $\pi / 2$ corners are allowed (no diagonal segments). This same classical result is shown again in Fig. 4(b) together with the result of the directedness density for a point in the middle of the Gaussian $(X Y)$ phase $\left(\mathcal{K}=0.5, \quad \mathcal{L}_{21}=-0.25, \quad \mathcal{L}_{22}\right.$ $=-1.0$, and $\mathcal{L}_{11}=0$ corresponding to $D=0$ and $J=-0.5$ ). A further discussion of the numerical results as well as the interpretation of the finite temperature behavior can be found in Appendix C.

Our general conclusion, based also on Monte Carlo studies of the behavior in other phases summarized in Appendix $\mathrm{C}$, is that apart from some extreme classical limits, the general lattice string model at zero temperatures is a directed string. The phase diagram of the general string model introduced in Sec. II will essentially be the same (apart from special limits) as the corresponding phase diagram of the simplified directed string model. In the remaining sections of this paper we will therefore focus on the phases and phase transitions of the directed string.

Although we have not found yet a formally rigorous description of the directedness symmetry breaking, we can offer a qualitative explanation at least on the level of our specific model. As we showed in Sec. III, the string problem can be mapped on the problem of two strongly coupled classical RSOS surface problems. The symmetry breakings of a single RSOS surface will be discussed in great detail later, but for the present discussion it suffices to know that such a single surface can be fully ordered, as well as (partly) disordered. Because of the strong coupling, it would a priori appear questionable to discuss the dynamics of the full model of Sec. II in terms of the dynamics of the two separate RSOS subproblems. However, in the context of directedness it is quite convenient to do so. When both the $x$ and $y$ RSOS problems would be fully disordered, it is easy to see that the string vacuum would be undirected. This is illustrated in Fig. 5(a): Two kinks moving the string from a $(1,0)$ to a $(0,1)$ direction in the lattice correspond to one kink that can move freely in the horizontal part of the string and one kink that can move freely in the vertical part of the string. On the other hand, when both RSOS problems are ordered, the string is also ordered. For instance, the $(1,0)$ string can be thought of as a combination of a RSOS surface that always steps upward in the $x$ direction and one that is horizontal in the $y$ direction [Fig. 5(b)].

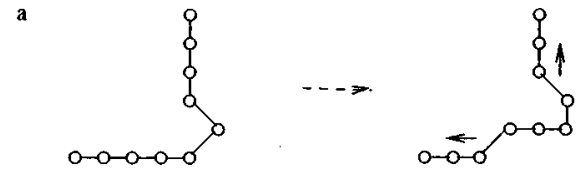

b

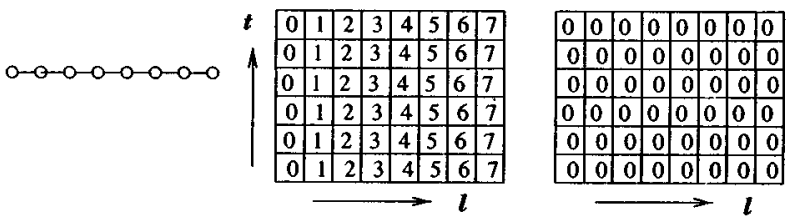

c

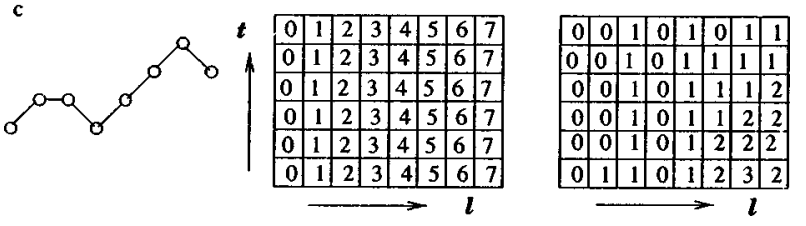

FIG. 5. (a) Undirected string with two kinks propagating along different directions. Note that the bend blocks the propagation of kinks. (b) $(1,0)$ string and the corresponding two (coupled) RSOS surfaces along the $x$ and the $y$ directions, respectively. The numbers correspond to the $x(y)$ position of hole $l$ at imaginary time $t$. (c) Disordered directed string and the corresponding ordered and disordered RSOS surfaces.

A third possibility is that one of the RSOS subproblems is ordered, while the other is disordered. Dismissing crumpled phases (such as condensates of the $\mathcal{L}_{11}$-type corners), the only possibility remaining is that one of the RSOS problems steps up always, while the other is disordered, as illustrated in Fig. 5(c). This results in a disordered directed string vacuum: The string steps always forward in, say, the $x$ direction, while it freely fluctuates in the $y$ direction. Hence the local order parameter underlying the directedness corresponds to the diagonal flat order (phase I of Fig. 8) of at least one of the two RSOS surfaces describing the string.

What is the source of the condensation energy? As we already stated, violation of directedness implies that $\pi / 2$ bends occur on the string, equivalent to overhangs on the world sheet. As can be easily seen, these bends block the propagation of links along the chain. Close to the bend itself the particles in the chain cannot move as freely as in the rest of the chain. This effect is shown in Fig. 6 .

Therefore, the presence of these bends increases the kinetic energy associated with the kink propagation and it makes no difference whether the bend consists of a single $\pi / 2$ corner or two $\pi / 4$ corners. This kinetic-energy cost dis-

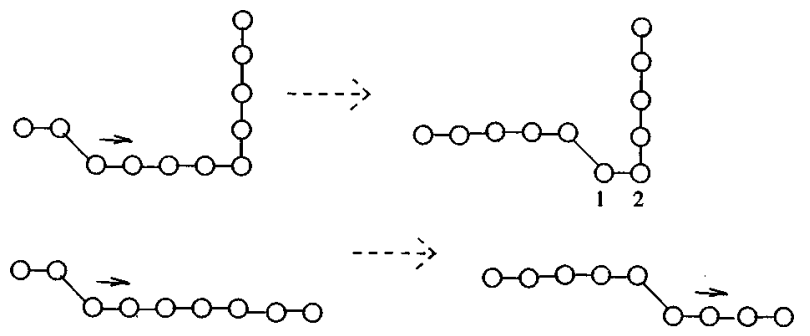

FIG. 6. Illustration of the fact that a bend blocks the propagation of links along the string. Note that holes 1 and 2 cannot move. 
appears when one of the two RSOS surfaces straightens and this drives the directedness condensation. It might be called a quantum order-out-of-disorder mechanism and it is suspected that a theory of the Hartree mean-field type can be formulated catching the phenomenon on a more quantitative level (with the kinks playing the role of electrons and the second surface offering the potentials). To emphasize the order-out-of-disorder aspect, it is easy to see that in the classical case, $\mathcal{T}=0$, in many regions of parameter space the problem becomes that of a self-avoiding walk on a lattice in the limit $T \rightarrow 0$, which does not exhibit the directedness order.

The directedness phenomenon might be viewed from a more general perspective. At zero temperature, the quantum string is equivalent to a thermally fluctuating sheet in three dimensions. Now it is well known from studies of classical interface ${ }^{34}$ that while a one-dimensional classical interface in two dimensions does not stay directed due to the strong fluctuations, for a two-dimensional sheet the entropic fluctuations are so small that interfaces can stay macroscopically flat in the presence of a lattice. ${ }^{35,36}$ For this reason, the roughening transition in a three-dimensional Ising model is properly described by (i.e., is in the same universality class as) a solid-on-solid model in which overhangs are neglected. ${ }^{35,36}$ In other words, even if microscopic configurations with overhangs are allowed, a classical interface on a lattice in three dimensions can stay macroscopically flat or "directed," in agreement with the findings from our specific model. Obviously, directedness order is rather fragile. It cannot exist at any finite temperature. When temperature is finite, the width of the world sheet in the imaginary time direction becomes finite as well and the long-wavelength fluctuations of the string becomes a 1D statistical problem, which cannot be directed.

\section{DIRECTED STRINGS AND THE SPIN-1 CHAIN}

Quite generally, the string problem does not simply reduce to that of the internal dynamics of the world sheet because of the requirement that the world sheet has to be embedded in $(D+1)$-dimensional space. However, in the presence of directedness order and in the absence of particle number fluctuations, ${ }^{21}$ the string boundary conditions are trivially fulfilled and the string problem is equivalent to that of a single "world sheet" in $1+1$ dimensions. Assume the string to be directed along the $x$ direction. Since the string steps always forward in this direction, the number of particles in the string has to be equal to the number of lattice sites in the $x$ direction and every directed string configuration will connect the boundaries in this direction. The string is still free to move along the $y$ direction. Instead of labeling the positions in the $2 \mathrm{D}$ plane the string is completely specified by the list of links, for which there are only three possibilities [in the $(1,1),(1,0)$, or $(1,-1)$ direction], and the position of a single "guider point." As a guider point we can take the position $\mathbf{r}$ of any one of the particles, which, together with the relative coordinates given by the links, fixes the position of the entire string. Since the guider represents just a single degree of freedom and since the thermodynamic behavior of a chain is determined by the link interactions, the guider coordinates will be irrelevant for the behavior of the

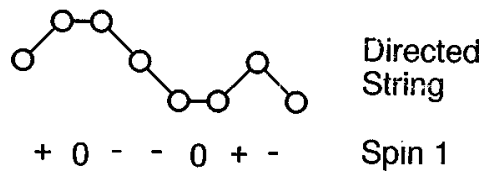

FIG. 7. Relation between spin 1 and directed strings, $S_{l}^{z}=y_{l+1}$ $-y_{l}$.

chain. Apart from this guider degree of freedom the directed string problem reduces to a one-dimensional quantum problem with three flavors.

From Eq. (3.5) one directly deduces the Hamiltonian of the string directed along $x$,

$$
\begin{aligned}
\mathcal{H}_{\mathrm{eff}}= & \sum_{l, k}\left[\frac{\mathcal{K}}{n} \delta\left(\left|y_{l+1, k}-y_{l, k}\right|-1\right)\right. \\
& +\frac{\mathcal{L}_{12}}{n} \delta\left(\left|y_{l+1, k}-y_{l-1, k}\right|-1\right) \\
& +\frac{\mathcal{L}_{22}}{n} \delta\left(\left|y_{l+1, k}-y_{l-1, k}\right|-2\right) \\
& \left.+\ln \left(\frac{\mathcal{T}}{n}\right) \delta\left(\left|y_{l, k}-y_{l, k+1}\right|-1\right)\right] .
\end{aligned}
$$

It is clear that the directedness simplifies the model considerable. The directed version can not self-intersect and the excluded-volume constraint is satisfied automatically. Furthermore, the $\mathcal{L}_{11}$ type of configurations are not allowed, thus the directed model is specified by three parameters and the temperature $(\mathcal{T}=1)$. Because of the preceding considerations, Eq. (5.1) corresponds to a $(1+1)$-dimensional problem, which is actually equivalent to a general quantum spin-1 chain.

We identify the spin with the string height difference $y_{l+1}-y_{l}$, which can be either 0,1 , or -1 ; see Fig. 7 . These link dynamical variables specifying the string can be directly identified with the $m_{s}=0, \pm 1$ variables of the spins on the sites of the spin chain. Defining the latter using hard-core bosons $b_{m_{s}}^{\dagger}$, the spin operators for the $S=1$ case become $S^{z}=b_{1}^{\dagger} b_{1}-b_{-1}^{\dagger} b_{-1}$ and $S^{+}=\sqrt{2}\left(b_{1}^{\dagger} b_{0}+b_{0}^{\dagger} b_{-1}\right)$ and by comparing the action of the spin and string operators on their respective Hilbert spaces one arrives at operator identities. ${ }^{26}$ A quantum hop from $y$ to $y+1$ increases the height difference on the left of $l$ by one and decreases it by one on the right, as is easily seen by inspecting the two hopping terms in Fig. 2. Therefore, ${ }^{26}$

$$
\begin{gathered}
S_{l}^{z}=y_{l+1}-y_{l}, \\
S_{l-1}^{ \pm} S_{l}^{\mp}=2 P_{\mathrm{str}}\left(y_{l}-y_{l-1}\right) P_{\mathrm{str}}\left(y_{l+1}-y_{l}\right) e^{ \pm i \hat{\pi}_{l}} .
\end{gathered}
$$

The identities, for $S=1$,

$$
\begin{gathered}
\delta\left(\left|y_{l+1}-y_{l}\right|-1\right)=\left(S_{l}^{z}\right)^{2} \\
\delta\left(\left|y_{l+1}-y_{l-1}\right|-1\right)=\left(S_{l}^{z}\right)^{2}+\left(S_{l-1}^{z}\right)^{2}-2\left(S_{l}^{z} S_{l-1}^{z}\right)^{2} \\
\delta\left(\left|y_{l+1}-y_{l-1}\right|-2\right)=\frac{1}{2} S_{l}^{z} S_{l-1}^{z}\left[1+S_{l}^{z} S_{l+1}^{z}\right]
\end{gathered}
$$


are easily checked. The directed string problem can now be reformulated in spin language as

$$
\begin{aligned}
\mathcal{H}_{\text {spin }}= & \sum_{l}\left[\left(\mathcal{K}+2 \mathcal{L}_{12}\right)\left(S_{l}^{z}\right)^{2}+\frac{\mathcal{L}_{22}}{2} S_{l}^{z} S_{l-1}^{z}\right. \\
& +\left(\frac{\mathcal{L}_{22}}{2}-2 \mathcal{L}_{12}\right)\left(S_{l}^{z} S_{l-1}^{z}\right)^{2} \\
& \left.+\frac{\mathcal{T}}{2}\left(S_{l}^{+} S_{l-1}^{-}+S_{l}^{-} S_{l-1}^{+}\right)\right]
\end{aligned}
$$

Following the spin-1 literature, we define the parameters

$$
\begin{gathered}
D=\mathcal{K}+2 \mathcal{L}_{12}, \\
J=\mathcal{L}_{22} / 2, \\
E=\mathcal{L}_{22} / 2-2 \mathcal{L}_{12} .
\end{gathered}
$$

The $E$ term is new. It is a quartic Ising term, leading to extra phases and phase transitions. For the special choice $E=0$ $(\mathcal{T}=1)$, the above Hamiltonian reduces to the familiar $X X Z$ model with on-site anisotropy,

$$
\mathcal{H}_{X X Z}=\sum_{l}\left[D\left(S_{l}^{z}\right)^{2}+J S_{l}^{z} S_{l-1}^{z}+\frac{1}{2}\left(S_{l}^{+} S_{l-1}^{-}+S_{l}^{-} S_{l-1}^{+}\right)\right]
$$

The zero-temperature phase diagram of the above spin-1 model has been discussed in detail in the literature. ${ }^{37-40,26}$ In Sec. VI we will briefly review the six phases found for this model, from a string perspective. Then we will show that a nonzero $E$ parameter leads to the appearance of four extra phases in Sec. VII.

den Nijs and Rommelse ${ }^{26}$ discuss a direct mapping between the spin chain and the RSOS surface. We stress that this mapping in fact involves two steps. First the RSOS model is mapped on a string problem, using the $T$ matrix. Then the spins are identified as shown above. Thus the quantum string is a natural intermediate of the two other models. den Nijs and Rommelse make use of the freedom in the choice of the $T$ matrix to define a mapping that is slightly different from ours since they introduce a transfer matrix along a diagonal, while we introduce one along the $x$ direction. As a result, in their case there are only interactions between next-nearest neighbors along the $(1,1)$ direction, while our choice allows for interactions between next-nearest neighbors along the $x$ direction. Therefore, our RSOS model differs slightly from theirs.

The RSOS representation is more transparent than the quantum model. The spin-1 phases and the nature of the phase transitions all have a natural interpretation in spacetime. For instance, the Haldane phase, or Affleck, Kennedy, Lieb, and Tasaki (AKLT) wave function, with its mysterious hidden string order parameter is identified as a "disordered flat', RSOS surface ${ }^{26}$ with a simple local order parameter. The height representation, dual to the spins, gives a similar local order parameter for the quantum string.

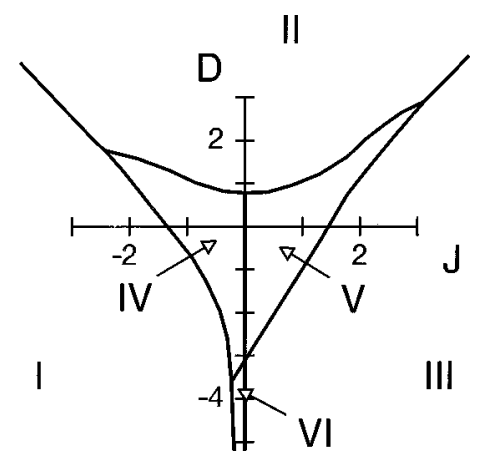

FIG. 8. Phases and phase transitions of the directed quantum string as a function of the on-site anisotropy $D$ and the Ising interaction $J$ of the corresponding spin- $1 X X Z$ model. The parameter $E$ is set to zero.

\section{PHASES $(E=0)$}

In this section the general string Hamiltonian will be simplified by leaving out the quartic Ising term $[E=0$ in Eqs. (5.4) and (5.5)]. Our string problem is now equal to the spin-1 $X X Z$ model. The zero-temperature phase diagram of the string problem is surprisingly rich, and even for the case $E=0$ there are six phases and a large variety of phase transitions. These phases can be classified in three groups: classical strings localized in space, quantum rough strings of the free variety, and partly delocalized phases of which the disordered flat phase is a remarkable example. In this section we will briefly review the six phases as discussed in the literature on the spin-1 $X X Z$ problem (5.6). The problem will be addressed from the quantum string perspective. For more details we refer to Ref. 26. In Sec. VII we will show that with a finite $E>0$ four additional phases are stabilized.

The phase diagram of the quantum string is shown in Fig. 8 as a function of $D$ and $J$. We have used the $X X Z$ model parameters, defined in Eq. (5.5), such that the phase diagram can be compared directly with the spin-1 literature ${ }^{37-40}$ and in particular with Fig. 13 of Ref. 26. We will introduce below the various order parameters that have been introduced in this reference to distinguish the six phases in this phase diagram. The relation between the more general $(E \neq 0)$ string and spin phases will be clarified in Sec. VII.

There is first of all a horizontal and a diagonal string phase. In the diagonal phase I no quantum fluctuations are allowed since a diagonal string does not couple to other states by $\mathcal{H}_{Q}$ (this is illustrated in Fig. 16 in Appendix A, to which we refer for further details). This phase is stabilized by a large and negative $\mathcal{L}_{22}$, so that since $E=0$ also $J$ $=\mathcal{L}_{22} / 2=2 \mathcal{L}_{12}$ is large and negative. A suitable variable introduced to define order parameters, following Ref. 26, is the Ising spin variable $\sigma_{l}=(-1)^{y_{l}}$, which identifies whether a given height is in an even or odd layer. This underlying spin model can have "ferromagnetic" or "antiferromagnetic" order and so we introduce the corresponding order parameters $^{41}$

$$
\begin{gathered}
\rho=\left\langle\sigma_{l}\right\rangle, \quad \rho_{\text {stag }}=\left\langle(-1)^{l} \sigma_{l}\right\rangle, \\
\rho_{\text {str }}=\left\langle\sigma_{l}\left(y_{l+1}-y_{l}\right)\right\rangle .
\end{gathered}
$$


Here the angular brackets denote the ground-state expectation value as well as an average over string members $l$. In Eq. (6.1) we have also included the order parameter $\rho_{\text {str }}$ discussed below. In the horizontal phase II one particular height is favored, thus the order parameter $\rho$ is nonzero here. This phase is stabilized by a large positive $\mathcal{K}$, which suppresses diagonal links. However, $\mathcal{H}_{Q}$ causes virtual transitions from two horizontal links into two diagonal ones; see Fig. 2. On the 2D world sheet these fluctuations show up as local terraces that do not overlap and thus do not destroy the longrange order. In both phases the elementary excitations are gapped.

Upon lowering $\mathcal{K}$ the terraces grow and at some point they will form a percolated network: The string has become disordered in both space and imaginary time. Via the wellknown Kosterlitz-Thouless roughening transition, ${ }^{35}$ phase IV is entered for $J<0$. This phase belongs to the well-known $X Y$ universality class, characterized by algebraic correlation functions and gapless meandering excitations: capillary waves in fluid interface language. The roughness, however, is extremely "soft" and the height difference diverges only logarithmically, $\left\langle\left(y_{l}-y_{m}\right)^{2}\right\rangle \sim \ln |l-m|$. The transition from the Gaussian phase that is rough and on average oriented horizontally to the "frozen" diagonal phase is a "quasi-firstorder" potassium dihydrogen phosphate (KDP) transition. ${ }^{26}$

For large negative $\mathcal{K}$ diagonal links are favored over horizontal ones. There is a transition to a second rough phase (phase VI). It is distinguished from the first by the order parameter $\rho_{\text {stag }}$, which is zero in phase IV. In this phase horizontal links are virtual and occur in pairs. As we will discuss later in Sec. VII, for large negative $K$ the model can therefore be reduced to an effective spin $-\frac{1}{2}$ problem.

For negative $\mathcal{K}$ and positive $J\left(=\mathcal{L}_{12} / 2=\mathcal{L}_{22} / 8\right)$ the string becomes a (physically unlikely) zigzag with alternating up and down diagonal pieces. Excitations to pairs of horizontal links are gapped. Again $\rho_{\text {stag }}=\left\langle(-1)^{-l} \sigma_{l}\right\rangle$ serves as an order parameter. Upon increasing $\mathcal{K}$ the islands formed by pairs of horizontal links start to overlap and there is an Ising transition into the Haldane or disordered flat (DOF) phase.

The point $J=1, D=0$ belongs to the gapped DOF phase, in agreement with Haldane's educated guess ${ }^{42,43}$ that integer spin chains are gapped at the Heisenberg antiferromagnetic point. In this "disordered horizontal" string phase the prototypical wave function, equal to the AKLT valence bond state, ${ }^{44}$ has every up diagonal link followed by a down link, with a random number of horizontal links in between. The height $y_{l}$ takes just two values, say, 0 and 1 . The local order parameter $\rho_{\text {str }}$ is defined in Eq. (6.1). This order parameter measures the correlation between the next step direction and whether one is in a layer of even or odd height. When $\rho_{\text {str }}$ $=1$, the string just steps up and down between two layers, but the steps can occur at arbitrary positions. Note that the height is a global quantity in spin language, i.e., it is the accumulated sum over spins $y_{l}=\sum_{m=0}^{l} S_{m}^{z}$. Because of this the above order parameter becomes nonlocal when rewritten in terms of the "string" of spins. Therefore, it is often called the string order parameter. We will also use this name, but stress that the "string of spins" to which this name refers should not be confused with the general strings that are the basis of our model and that the other order parameters are nonlocal as well in terms of the original spins $S$.

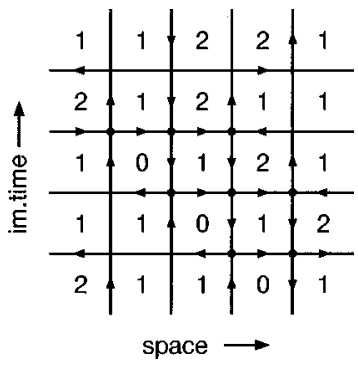

FIG. 9. Vertices (thick dots) on the space, imaginary-time string world sheet. The numbers correspond to the heights $y_{l, k}$. Arrows are drawn when the heights of neighbors differ. When four arrows occur at a crossing point this is called a vertex.

This phase diagram can be rationalized by writing the RSOS problem as the product of a six-vertex model and the 2D Ising model of $s$ spins on the six-vertex lattice, as discussed in detail by den Nijs and Rommelse. ${ }^{26}$ The horizontal, diagonal, zigzag, and also the second rough phase VI all correspond to Ising order: $\rho=\left\langle\sigma_{l}\right\rangle$ is nonzero in the horizontal phase II, while $\rho_{\text {stag }}=\left\langle(-1)^{l} \sigma_{l}\right\rangle$ is nonzero in the diagonal phase I, the zigzag phase III, and the rough phase VI. The six-vertex part is defined on the crossing points of steps on the surface; see Fig. 9. This is a (sometimes highly) diluted set of points. The Ising degree of freedom disorders on the transition between phases III and V and between IV and VI, while the six-vertex part remains unchanged. Therefore, these transitions are Ising like. Transitions $\mathrm{I} \rightarrow \mathrm{IV}, \mathrm{I} \rightarrow \mathrm{VI}$, $\mathrm{IV} \rightarrow \mathrm{V}$, and $\mathrm{III} \rightarrow \mathrm{VI}$ are related to the six-vertex part becoming critical and these KDP and Kosterlitz-Thouless (KT) transitions are known from the quantum spin- $\frac{1}{2}$ chain. The transition II $\rightarrow \mathrm{IV}$ is related to the famous surface-roughening transition, of the Kosterlitz-Thouless type. ${ }^{35,36}$ The subtle transition between phase II and V is coined a "preroughening transition" by den Nijs. It separates two gapped phases. At the transition the gap closes and the system is Gaussian, with varying exponents along the transition line. ${ }^{37-40}$

Almost all the phases can be distinguished by the above order parameters $\rho, \rho_{\text {stag }}$, and $\rho_{\text {str }}$, except that these do not discriminate between the diagonal phase I and the rough phase VI. These two phases can be identified by also introducing an order parameter that detects the presence of an average slope $p_{\text {slope }}=\left\langle y_{l+1}-y_{l}\right\rangle$. In Table I we list the various phases for $E=0$ and the order parameters.

As we shall see in the next section, in the general case $E \neq 0$ it is more convenient to introduce slightly different spin variables to identify all the ten different phases that

TABLE I. Order parameters that distinguish between the six different phases in the phase diagram for $E=0$. A plus entry in the table indicates that the particular order parameter is nonzero.

\begin{tabular}{ccccc}
\hline \hline Phase & $\rho$ & $\rho_{\text {stag }}$ & $\rho_{\text {str }}$ & $\rho_{\text {slope }}$ \\
\hline I & & + & & + \\
II & + & & & \\
III & & + & + & \\
IV & & & & \\
V & & & + & \\
VI & & + & & \\
\hline \hline
\end{tabular}




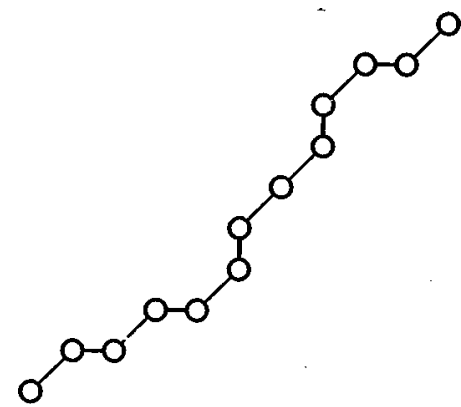

FIG. 10. Typical low-temperature string in the slanted parameter region VII.

occur then. The choice of Ref. 26 discussed here is somewhat more convenient for understanding the universality classes of the various phase transitions.

\section{THE FULL PHASE DIAGRAM: PHASE-BOUNDARY ESTIMATES}

As mentioned above, the quartic Ising term with prefactor $E$ generalizes the $X X Z$ Hamiltonian and leads to extra phases. We will show that four extra phases are to be expected and that they are stabilized by a positive $E$ parameter. The most disordered phase is still the Gaussian phase (see Fig. 10).

Using a decomposition similar to that above, we can determine how many different phases to expect for a general spin-1 chain with z-axis anisotropy and nearest-neighbor interactions. ${ }^{45}$ Think of the spin 1 as consisting of two spins $\frac{1}{2}$; see Table II. The first is $\sigma^{z}=\downarrow$ when the spin 1 has $S^{z}$ $=0$ and $\sigma^{z}=\uparrow$ when $S^{z}= \pm 1$, similarly to the Ising degree of freedom defined above. This spin thus indicates the presence or absence of a step. The second spin $\frac{1}{2} \mathbf{S}$ is defined as $s^{z}$ $=S^{z} / 2$ when $S^{z}= \pm 1$ and is absent when $S^{z}=0$. This is related to the diluted vertex network discussed by den Nijs and Rommelse, in that if there is a step, the $z$ component of $\mathbf{s}$ indicates whether this step is up or down. The spins $\mathbf{s}$ can have ferromagnetic $(F)$ or antiferromagnetic (AF) order or they can be disordered $(D)$. For $\sigma$ the two ferromagnetic cases correspond to different physical situations and we have to distinguish ferromagnetic $\downarrow$ (F2), a horizontal string, from ferromagnetic $\uparrow(F 1)$. When $\sigma$ has $F 2$ order, $\mathbf{s}$ becomes irrelevant (or better, there are disconnected finite terraces of $\mathbf{s}$ spins with short-range correlations). Therefore, one expects ten phases, depending on the order of the two spin species: one $F 2$ phase, three $F 1$ phases, three $\sigma$-disordered phases, and three $\sigma$-antiferromagnetically ordered phases. These are listed in Table III. An example of a phase diagram in a case in which all ten phases are present is show in Fig. 11 , which corresponds to the case $E=5$. The detailed of how this phase diagram was obtained will be discussed below.

TABLE II. Spin-1 $S$ seen as a combination of two spins $\frac{1}{2}, \sigma$ and

\begin{tabular}{cccc}
\hline \hline$S$ & 1 & 0 & -1 \\
\hline$\sigma$ & $\uparrow$ & $\downarrow$ & $\uparrow$ \\
$s$ & $\uparrow$ & & $\downarrow$ \\
\hline \hline
\end{tabular}

TABLE III. Schematic representation of the different phases. Also shown is the long-range order of the two spins $\frac{1}{2}, s$ and $\sigma$, as defined in the text. $F$ denotes ferromagnetic, $F 1$ up-spin ferromagnetic, $F 2$ down-spin ferromagnetic, AF antiferromagnetic, and $D$ disordered.

\begin{tabular}{|c|c|c|c|c|}
\hline Phase & $\sigma$ & s & String & Spin 1 \\
\hline I & F1 & $\mathrm{F}$ & & ++++++++ \\
\hline II & F2 & & & $\begin{array}{llllllll}0 & 0 & 0 & 0 & 0 & 0 & 0 & 0\end{array}$ \\
\hline III & F1 & $\mathrm{AF}$ & & +-+-+-+- \\
\hline IV & D & D & $\because$ & $+0-+0+0-+$ \\
\hline V & D & $\mathrm{AF}$ & & $-+0-00+-$ \\
\hline VI & $\mathrm{F} 1$ & D & & +-++-+-- \\
\hline VII & D & $\mathrm{F}$ & & $0+0++0+00$ \\
\hline VIII & $\mathrm{AF}$ & $\mathrm{F}$ &.$^{\bullet}$ & $0+0+0+0+0$ \\
\hline IX & $\mathrm{AF}$ & D & & $0+0+0-0+0$ \\
\hline $\mathrm{X}$ & $\mathrm{AF}$ & $\mathrm{AF}$ &.$\bullet^{\bullet \cdot} \cdot$ & $0+0-0+0-0$ \\
\hline
\end{tabular}

There are four new phases, VII-X, compared to the phase diagram discussed in Sec. VI. All four are stabilized by a positive $E$ parameter in Eq. (5.4). Three phases, VIII-X, result from an antiferromagnetic order of the $\sigma$ spin. This corresponds to alternating horizontal and diagonal string links (see Table III). The diagonal links can be either all up [ferromagnetic (FM), phase VIII], alternatingly up and down (AF, phase X), or disordered (phase IX). In phase VII the $\sigma$ spin is disordered, while the s spin is in the FM phase. This is a diagonal wall diluted with horizontal links. These links coherently move up and down along the wall, lowering the kinetic energy. The wall can take any average angle between $-\pi / 4$ and $\pi / 4$ and this angle is fixed by the value of the

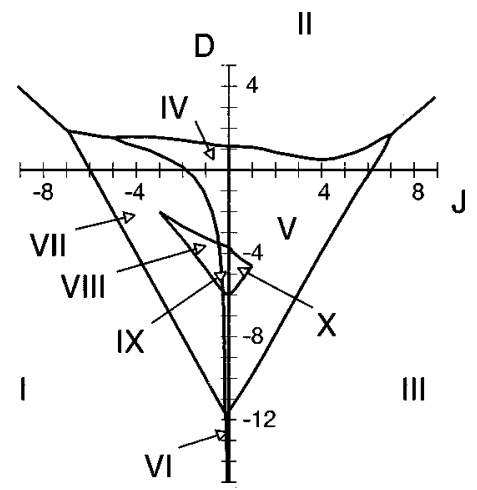

FIG. 11. Phases and phase transitions of the quantum string for $E=5$ as a function of $t$. On the axis are the on-site anisotropy $D$ and the Ising interaction $J$. 
parameters. We will call this the "slanted" phase. In terms of the decomposition into an Ising spin model and a sixvertex model of den Nijs and Rommelse it is easy to see that the horizontal links change the orientation of the Ising spin and act like a Bloch wall. The Ising spin is therefore disordered. The six-vertex term is irrelevant for the existence of the slanted phase: In the case of a single horizontal link, i.e., on the boundary between the slanted and diagonal string phase, there are no vertices.

A large part of the phase boundaries can be estimated exactly, almost exactly, or to a fair approximation. Let us focus first on the classical phases. The diagonal, horizontal, and zigzag phases have the following energies in the classical approximation in which there are no fluctuations, as is easily verified:

$$
\begin{gathered}
E_{\mathrm{I}}=L\left(\mathcal{K}+\mathcal{L}_{22}\right)=L(D+J+E), \\
E_{\mathrm{II}} \approx 0, \\
E_{\mathrm{III}} \approx L \mathcal{K}=L(D-J+E),
\end{gathered}
$$

where $L$ is the length of the chain. The first-order transitions will therefore occur close to the lines $\mathcal{K}=-\mathcal{L}_{22}(D=-J$ $-E)$ between phases I and II, $\mathcal{L}_{22}=0 \quad(J=0)$ between phases I and III, and $\mathcal{K}=0(D=J-E)$ between phases II and III. These transitions become exact in the classical or large-spin limit.

The transition between phases I and VII, the diagonal and slanted phases, can be found exactly. The transition is of the Pokrovsky-Talapov or conventional 1D metal-insulator type (see, for instance, Ref. 25). The horizontal link can be seen as a hard-core particle or a spinless fermion, with the parameters determining an effective chemical potential. For a critical chemical potential equal to the bottom of the band of the hard-core particle the band will start to fill up. The transition occurs when the diagonal string becomes unstable with respect to a diagonal string with one horizontal link added. This single link delocalizes along the string with a momentum $k$ and a kinetic energy $2 \mathcal{T} \cos (k)$. The minimal energy is $(L-1) \mathcal{K}+(L-2) \mathcal{L}_{22}+2 \mathcal{L}_{12}-2 \mathcal{T}$ and the transition occurs when $\mathcal{K}=2\left(\mathcal{L}_{12}-\mathcal{L}_{22}-\mathcal{T}\right)$ or, with $\mathcal{T}=1$, when the phase I to phase VII transition

$$
D=-2(J+E+1)
$$

occurs.

The transition between phases III and V will occur when horizontal link pairs unbind in the zigzag background. A rough estimate, neglecting fluctuations, is obtained by comparing the energy of a single horizontal link with that of a perfect zigzag. In the same way as above we estimate the phase boundary to be close to $D=2(J-E-1)$. In the same way the transition from phase II to V or IV is determined by the energy of a single diagonal step in a horizontal wall, which becomes favorable when $D=2$. This last estimate turns out to be very crude, in that it largely underestimates the stability of the flat phase.

For large negative $\mathcal{K}$ the horizontal links are strongly suppressed and the string can be mapped perturbatively on a spin- $\frac{1}{2}$ chain. Identify $S^{z}=1$ (diagonal upward) with $s^{z}=\uparrow$ and $S^{z}=-1$ (diagonal downward) with $s^{z}=\downarrow$. Via a virtual
$(0,0)$ spin pair (two horizontal links) the spins can still fluctuate, $(1,-1) \rightarrow(0,0) \rightarrow(-1,1)$. One finds, using secondorder perturbation theory in $\mathcal{T} / \mathcal{K}$,

$$
\begin{aligned}
\mathcal{H}_{\text {eff }}(D \rightarrow-\infty)= & \left(4 J+j_{ \pm}\right) \sum_{l} s_{l}^{z} s_{l+1}^{z} \\
& +j_{ \pm} \frac{1}{2} \sum_{l}\left(s_{l}^{+} s_{l+1}^{-}+s_{l}^{-} s_{l+1}^{+}\right), \\
j_{ \pm} & =\frac{2 \mathcal{T}^{2}}{|2 D+3 E|} .
\end{aligned}
$$

Here we subtracted an irrelevant constant term. This has the form of the well-studied spin- $\frac{1}{2}$ Heisenberg chain with Ising anisotropy. Transitions occur when $4 J+j_{ \pm}= \pm j_{ \pm}$or when $J=0$ (III to VI) and $J=-1 /|2 D+3 E|$ (I to VI) (setting $\mathcal{T}$ $=1$ ).

The above estimates seem to suggest that the line $J=0$ is special. Our numerical results show that it describes accurately the transition between phases III and VI, but also the transition between phases IV and V. This agrees with the arguments given by den Nijs and Rommelse ${ }^{26}$ that the Kosterlitz-Thouless transition between phases IV and V should occur precisely at the $J=0$ line.

The slanted phase consists predominantly of up diagonal and horizontal links. Neglecting down diagonals altogether, which turns out to be a good approximation, one can again map the string or spin- 1 chain on an effective spin- $\frac{1}{2}$ system. Now the relevant degree of freedom is the $\sigma$ Ising degree of freedom. Because $\sigma=\uparrow$ (a diagonal link) is not symmetrically equivalent to $\sigma=\downarrow$ (a horizontal link) the spins will "feel" an effective magnetic field, which regulates the density of horizontal links. Rewriting Eq. (5.4) gives

$$
\begin{aligned}
\mathcal{H}_{\mathrm{eff}}= & D \sum_{l}\left(\sigma_{l}^{z}+\frac{1}{2}\right)+(J+E) \sum_{l}\left(\sigma_{l}^{z}+\frac{1}{2}\right)\left(\sigma_{l+1}^{z}+\frac{1}{2}\right) \\
& +\mathcal{T} \sum_{l}\left(\sigma_{l}^{+} \sigma_{l+1}^{-}+\sigma_{l}^{-} \sigma_{l+1}^{+},\right)
\end{aligned}
$$

and, after rescaling and putting $\mathcal{T}=1$,

$$
\mathcal{H}_{\mathrm{eff}}=h \sum_{l} \sigma_{l}^{z}+\Delta \sum_{l} \sigma_{l}^{z} \sigma_{l+1}^{z}+\frac{1}{2} \sum_{l}\left(\sigma_{l}^{+} \sigma_{l+1}^{-}+\sigma_{l}^{-} \sigma_{l+1}^{+}\right) \text {, }
$$

with the field $h=(D+J+E) / 2$ and Ising coupling $\Delta=(J$ $+E) / 2$. On the line $h=0$ the number of up diagonal links equals the number of horizontal links. The average tilt angle is thus $22.5^{\circ}$ in this approximation. The phase diagram of the spin $-\frac{1}{2}$ chain in the $h-\Delta$ plane was discussed by Johnson and McCoy. ${ }^{46}$ For $h=0$ there are three phases. The ferromagnet corresponds to phase I, the antiferromagnet to phase VIII: and the gapless disordered phase translates to the slanted string phase VII. Increasing the field $h$ in the AF phase will cause a transition to the gapless phase with a finite magnetization. In the approximation that down diagonals are neglected, it follows from the results of Johnson and McCoy ${ }^{46}$ that the point $\Delta=1, h=0$ or $J=2-E$ is the point with the most negative value of $J$ where phase VIII is stable. For $E$ $=0$ (as well as for small values of $E$ ) this occurs in the 


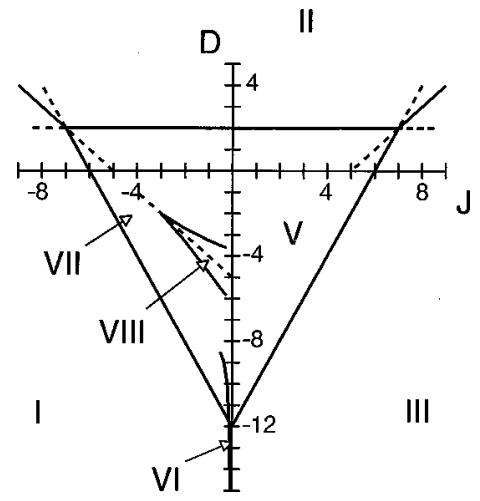

FIG. 12. Various phase transitions, obtained from semiclassical estimates, exact arguments, and perturbative mappings to spin $\frac{1}{2}$.

positive $J$ side of the phase diagram, meaning that the transition from phase VIII to phase $\mathrm{X}$ will in fact not be stable: For positive values of $J$, down steps in the original model proliferate. To have a phase diagram with all ten phases present we choose $E=5$.

In Fig. 12 the various phase-boundary estimates given above are summarized. The topology of the main part of the phase diagram has now become clear. In the center of the figure for $E=5$ the Johnson-McCoy phase diagram is inserted. The estimates suggest that at least phases VII and VIII are stabilized by taking $E=5$. The dotted line through phase VIII is the line where the effective field $h$ is zero and the number of diagonal links is (nearly) equal to the number of horizontal ones.

We finally argue that the slanted phase exists in some region of the phase diagram for any $E>0$. To see this, first consider the case $E=0$. Along the line $D=0$, our model (with $E=0$ ) corresponds to the Heisenberg model with Ising anisotropy and the point $J=-1$ corresponds to the isotropic ferromagnetic Heisenberg point. Along the line $D=0$, the transition from the "ferromagnetic" (diagonal in string language) phase I to the $X Y$ phase IV therefore occurs at $J$ $=-1$. Now, for $E=0$, the exact location of the line along which phase I becomes unstable to the slanted phase VII, given by $D=-2(J+1)$ according to Eq. (7.2), goes exactly through the ferromagnetic Heisenberg point at $J=-1$ as well. The results of Fig. 13 of den Nijs and Rommelse ${ }^{26}$ indicate that this line then touches the phase boundary between phases I and IV right at this point in such a way that for $E=0$ no slanted phase occurs. If we assume that both phase boundaries shift linear in $E$ for $E$ nonzero and small, it is clear that the slanted phase must stabilize in some region near the point $D=0, J=-1$. for one sign of $E$, while for the other sign the phase must be absent. Physically, it is clear that the stabilization of the slanted phase will occur for positive values of $E, E>0$.

\section{NUMERICAL ANALYSIS}

To fill in the details of the phase diagram we have performed exact diagonalization and finite-temperature quantum Monte Carlo calculations. Ground-state properties of strings up to 15 holes (spin chains of length 14) were obtained using the Lanczos diagonalization method. For the Monte Carlo method we used the checkerboard decomposition, briefly ex-

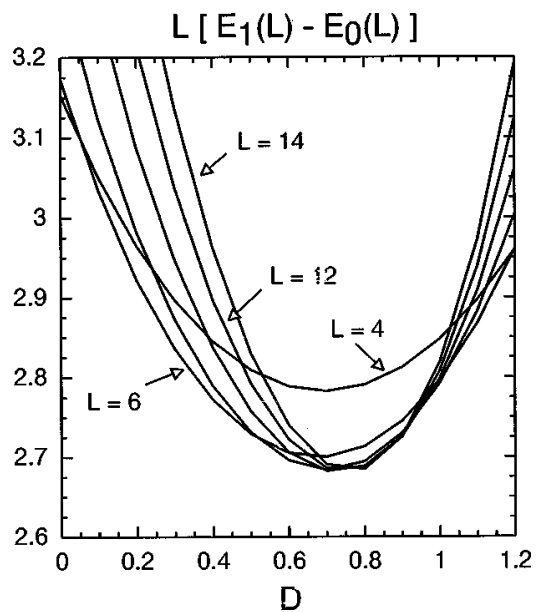

FIG. 13. Estimate of the preroughening transition between phases II and V. The plot shows $L\left[E_{1}(L)-E_{0}(L)\right]$ for various lengths $L$, as a function of the parameter $D$, with $J=0.8$ and $E$ $=0$. The two crossing points between the successive curves form an upper and lower estimate of the transition.

plained in Sec. III. The Monte Carlo method has the disadvantage that an extra limit to zero temperature has to be taken, a regime where the updating slows down considerably and where it is difficult to judge the accuracy. To determine the phase boundaries of the directed string we mainly used the Lanczos results for the equivalent spin model. On the other hand, the Monte Carlo space-time world sheets provide a transparent physical insight into the phases, phase transitions, order parameters, etc. Moreover, the Monte Carlo method allows, of course, one to treat bigger systems.

We are in the fortunate situation that the order parameters of the various phases and the universality classes of the transitions are known. This offers a variety of approaches to determine the critical lines: One can monitor the finite-size behavior of the order parameter, correlation functions, or the energy-level spacings. Typically we applied two independent methods to the various transitions. Our aim is to map out the entire phase diagram with an accuracy of roughly the line thickness in the phase diagram. For very accurate estimates other methods, notably the density-matrix renormalizationgroup treatment of White, ${ }^{47}$ are more appropriate.

An elegant and powerful method is the phenomenological renormalization-group approach pioneered by Nightingale. ${ }^{48,49}$ In this approach one considers an infinite strip with a width $L$, as a finite-size approximation to the 2D classical system. At the critical temperature of the infinite system one expects, from finite-size scaling, that the correlation length along the strip scales like the width of the strip $\left(L_{1}\right.$ or $\left.L_{2}\right)$,

$$
\xi_{L_{1}}\left(T_{c}\right)=\frac{L_{1}}{L_{2}} \xi_{L_{2}}\left(T_{c}\right)
$$

The infinite strip is solved by diagonalizing the (finite) $T$ matrix. The correlation length can be calculated from

$$
\xi=1 / \ln \left(\lambda_{1} / \lambda_{0}\right),
$$

where $\lambda_{0}$ and $\lambda_{1}$ are the largest and second largest eigenvalues of the $T$ matrix. 
A finite 1D quantum chain can be viewed as a strip infinitely long in the imaginary-time direction (zero temperature). In the time continuum limit, writing $T=\exp (\tau H)$, the equation corresponding to Eqs. (8.1) and (8.2) is

$$
L_{1}\left[E_{1}\left(L_{1}\right)-E_{0}\left(L_{1}\right)\right]=L_{2}\left[E_{1}\left(L_{2}\right)-E_{0}\left(L_{2}\right)\right]
$$

for two different string lengths $L_{1}, L_{2} \rightarrow \infty$ and for parameter values at criticality. Here $E_{0}$ and $E_{1}$ are the ground state and first excited energy of a quantum Hamiltonian $H$. According to Eq. (8.3), a phase transition line can be located by studying the energy gap as a function of $L$, while monitoring when Eq. (8.3) is obeyed. A practical example of Eq. (8.3) is shown in Fig. 13. Successive curves for lengths $L$ and $L$ +2 show two crossing points. Extrapolating these crossing points to infinite $L$ gives two estimates of the preroughening transition from phase II to phase V.

The above scaling holds when time and space scale in the same way or $E_{1}(q)-E_{0} \sim q^{z}$, with a dynamical exponent $z$ $=1$. This can be checked independently, giving a selfconsistent justification of the use of Eq. (8.3). In a similar spirit one can determine the critical scaling of correlation functions of an operator $O$ by monitoring the lowest-energy state with a nonzero overlap with $O|0\rangle$. This has been used extensively ${ }^{37-40}$ to study the phases and exponents of the Gaussian phases of the spin-1 chain. We refer to these articles for more details.

Another method used to determine second-order phase transitions is the Binder parameter, ${ }^{50}$ which we define as ( 3 $\left.-\left\langle m^{4}\right\rangle /\left\langle m^{2}\right\rangle^{2}\right) / 2$, where $m$ is the relevant order parameter. This quantity tends to 1 in the ordered phase, where $\left\langle m^{4}\right\rangle$ $\approx\langle m\rangle^{4}$, and approaches 0 in the disordered phase, where $m$ has a Gaussian distribution around $\langle m\rangle=0$. In a renormalization-group sense the shape of the order parameter distribution function becomes independent of the size at criticality. The various curves for different sizes of the Binder parameter versus model parameters or $T$ should therefore cross at the critical point. For instance, for the Ising transition between phases III and V we take $m=(-1)^{y_{l}-l}$, the Ising degree of freedom introduced before.

The Kosterlitz-Thouless transition between, for instance, phases IV and V is a subtle one due to the infinite order of the transition and the exponential vanishing of the gap. Previous studies show a large uncertainty in the position of the transition line. In the entire Gaussian rough string phase the system is critical. Height correlations diverge very weakly like $G(r)=\left\langle\left(y_{r}-y_{0}\right)^{2}\right\rangle \propto C \ln (r)$. At the KT transition point the prefactor takes the universal ${ }^{36}$ value $C=2 / \pi^{2}$. We found that this relation is very useful in determining the KT transition line; see Fig. 14. This relation gives surprisingly good results even for very small distances and is consistent with a KT transition at $J=0$, as discussed by den Nijs and Rommelse. ${ }^{26}$

The complete phase diagram for $E=5$ was already shown in Fig. 11. For this value of $E$ the slanted phase VII is very pronounced. Phases VIII-X occur in a small region in the middle of the diagram around the line of equal probability of horizontal and diagonal links, in the approximation that the Hamiltonian can be mapped onto the spin- $\frac{1}{2}$ problem (7.5). The rough phases occur at small negative values of $J$. Phases I-III, V, VIII, and X are gapped.

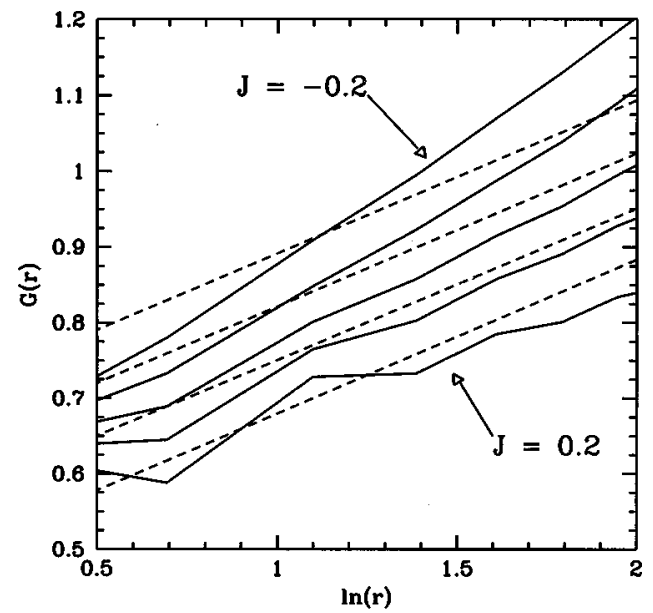

FIG. 14. Estimate of the Kosterlitz-Thouless transition between phases IV and V. At the transition the slope between $G(r)=\left\langle\left(y_{r}\right.\right.$ $\left.\left.-y_{0}\right)^{2}\right\rangle$ and $\ln (r)$ approaches $2 / \pi^{2}$ (the dotted lines).

The character of the various phases becomes clear by looking at the corresponding Monte Carlo snapshots in Fig. 15. In the flat phase one particular height $y_{l}$ dominates and the quantum fluctuations do not percolate in space-time. Decreasing $\mathcal{K}$ these quantum fluctuation islands grow and when they overlap the string enters phase IV. The system is Gaussian rough in both space and time. Note the very weak logarithmic meandering; e.g., for a string of length 10, Fig. 14 shows that the mean-square height fluctuations are only of order 1 near the KT transition. Despite this, determining the KT transition from $G(r)$ works surprisingly well for the small systems calculated.

The fascinating order parameter of the Haldane phase or disordered flat phase becomes transparent when looking at the world sheet, using the height representation instead of the spin-1 language. Globally, the surface is limited to two heights only and is therefore macroscopically flat. However in both the time and space directions there is a disordered array of up and down steps, with the restriction that every step up is followed by a step down, when the order is perfect. On flat pieces, however, there are local fluctuations (with consecutive up steps or down steps) decreasing the value of the order parameter. These islands will grow when $J \rightarrow 0$ from the positive side and when they overlap the string becomes rough. Note that the rigidity (flatness) is clear when viewing the overall structure of the world sheet in $1+1$ dimensions. On the other hand, from a single time slice one might be tempted to conclude that the string is rough.

The transition from phase I to phase VII is of the Prokovsky-Talapov type. Such transitions are often discussed in the context of a commensurate-incommensurate transition of a monolayer of atoms on a substrate with a different lattice parameter. In the "floating solid" phase such a system consists of a set of parallel domain walls with entropic meandering. ${ }^{25}$ The similarity to phase VII, illustrated in Fig. 15, is clear. The entropic meandering in the 2D classical case is now to be interpreted as the quantum motion of hard-core particles (horizontal links) along the string.

\section{DISCUSSION AND CONCLUSIONS}

Motivated by stripes, we have introduced a lattice string model for quantum domain walls and mapped out its full 


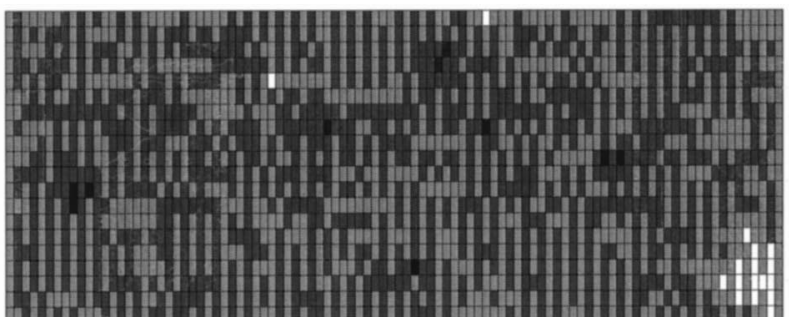

(a)

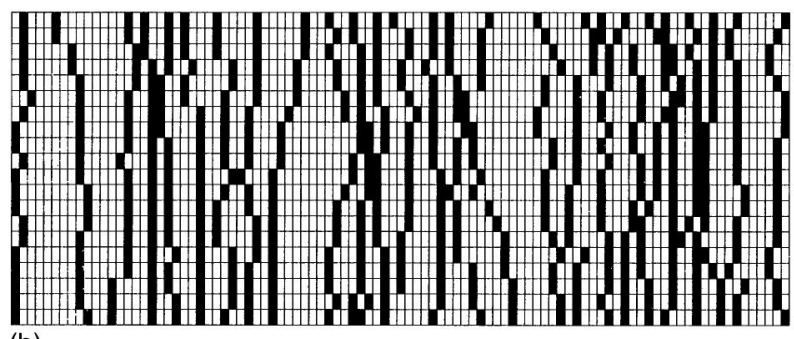

(b)

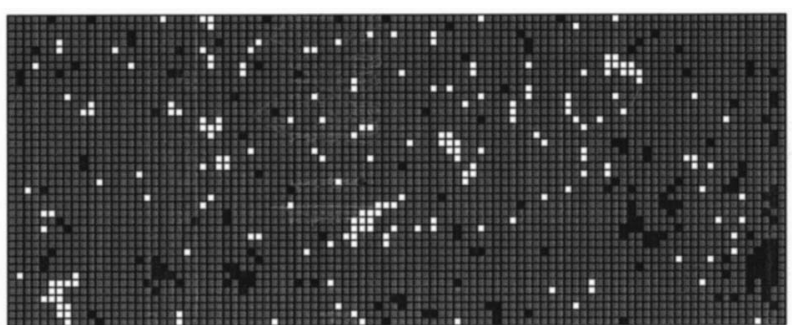

(c)

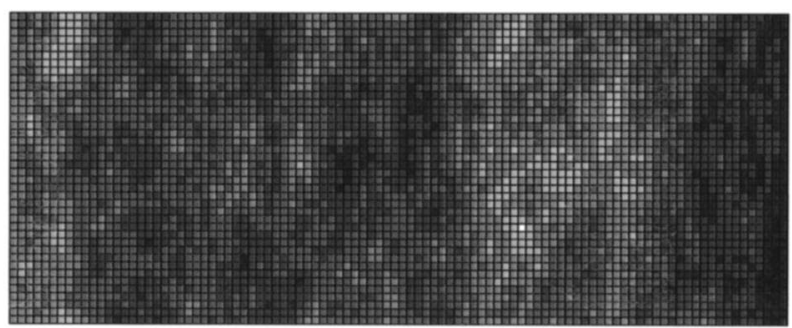

(d)

FIG. 15. Monte Carlo snapshots of (a) the Haldane phase (phase V, with $D=0, J=1$, and $E=0$ ), (b) the slanted phase (phase VII, with $D=-0.75, J=-5, E=5$ ), (c) the flat phase (phase II, with $D=2, J=0$, and $E=0$ ), and (d) the rough phase (phase IV, with $D=0, J$ $=-0.5$, and $E=0)$. Black to white means increasing height, except for the slanted phase in (d), where black denotes a horizontal link and white an up diagonal link.

phase diagram. We find a generic zero-temperature symmetry breaking: The string acquires a direction in all cases. The main reason is that bends in the string prohibit the quantum transport or, vice versa, the quantum motion of kinks straightens out the string (the "garden hose" effect of Nayak and Wilczek ${ }^{12}$ ). We arrive at the counterintuitive conclusion that for increasing kink quantum disorder the orientational preference of the string grows. The directed string problem that remains appears to be related to a well understood surface statistical physics (RSOS) model and simultaneously to a $S=1 X X Z$ quantum spin chain with single-site anisotropy. Motivated by the string interpretation, we found a number of phases described by this class of models that were previously not identified.

Physically, the phases fall in three main categories: classical (flat world sheet), Gaussian (rough world sheet), and disordered flat phases. The phases are further distinguished by the direction they take in the embedding space. Besides the flat strings in the horizontal and diagonal directions, we find that the disordered flat phases show here a rich behavior. Apart from the known phase with horizontal direction, which is associated with the incompressible phase of the spin model, we identified another category of disordered flat phases that take, depending on parameters, arbitrary directions in space (the slanted phases).

Although this does not apply to the localized strings, we suspect that a strong universality principle might apply to the delocalized strings: At least away from the phase boundaries to the localized phases, the underlying lattice renders the delocalized strings to be described by free field theory. The reason is simple: Regardless of the terms that one adds to the lattice scale action, the problem remains of the $X X Z$ kind and the massless phases fall into the $(1+1)$-dimensional $\mathrm{O}(2)$ universality class. For instance, one can add other kinkkink interactions, etc., and these can be all described by products of $S^{z}$ operators. Although these operators determine the nature of the localized phases, they turn into irrelevant operators in the massless phases. The kinetic sector is more subtle. For instance, one would like to release the constraint that kinks only occur with "height flavor' \pm 1 . This means in surface language that one partially lifts the restrictedness of the RSOS model or in spin language that one increases the total spin; e.g., $S=2$ means in string language that kinks occur describing height differences of \pm 2 as well. Although increasing the magnitude of spin has an influence on the localized phases, it does not change the fact that the massless phase away from the phase boundaries is still obeying $X Y$ universality. A point of caution is that the holes in principle could change their order when larger excursions are allowed. However, these "exchange loops" are strictly local and therefore irrelevant for the long-wavelength behavior as long as the string is internally an insulator. These could represent more of a problem for strings that are internally superconductors or metals.

We also stress that it follows from the arguments of den Nijs and Rommelse ${ }^{26}$ that the occurrence of a gapped Haldane-type phase for strings is not a peculiar feature of the spin-1 representation, but a general consequence of the existence of further neighbor interactions between the holes in strings.

Do our findings bear any relevance to the stripes in cuprates? At the very least, they do bring up some interesting questions.

(a) Is the stripe solidification in, for instance, the LTT cuprates $^{3}$ initially driven by a single string effect or by a collective transition of the string liquid? In the end it has to be the latter since a single string cannot undergo phase transitions at finite temperatures. However, it can be well imagined that the effect of the LTT-pinning potential is to stabilize $(1,0)$ directed stripes over $(1,1)$ stripes. In the language of this paper, this amounts to an increase of the parameter $K$, which could move the stripe from the Gaussian phase into the horizontal flat phase. At zero temperature, this would turn individual stripes in straight rods that are obviously 
much easier to order than meandering strings. At finite temperatures, this could increase the single-string persistence length substantially, so that stripe-stripe interactions become more effective in stabilizing a stripe solid at finite temperatures. ${ }^{51}$ Further work is needed to establish if these single-stripe transitions are of relevance.

(b) Do the disordered flat string phases exist? The simplest disordered flat phase is the horizontal one (phase V) corresponding with the Haldane phase of the $S=1$ spin chain. In string language, this is nothing but a localized string along the $(1,0)$ direction in the lattice, which is, however, not site centered (as is phase II) but, on average, bond centered. Bond-centered stripes show up in the numerical study of the $t-J$ model by White and Scalapino, ${ }^{19}$ which shows that the ground state of this model at finite dopings is a stripe phase. A main difference with the mean-field stripes is that these $t-J$ stripes are bond centered. Initially, one could be tempted to think that this has a truly microscopic reason: Charges in $t-J$ tend to be on links. However, it could also be due to a collective string effect: It could be "our" phase V. This can be easily established by measuring the appropriate (string) correlators. Is it the case that on equal times the charges are on sites while the kinks take care of delocalizing the stripes over two lattice rows or is it so that on all times the charges are on the links? This is obviously an important question in the light of recent works relating the bond centering via Hubbard-ladder physics to superconductivity. ${ }^{22}$ We also notice that there are experimental indications for bond centering in the nickelates ${ }^{52}$ where disordered flatness could possibly also play a role.

(c) If well developed stripes exist in the superconductors and/or metals, these have to occur in the form of a quantum disordered stripe phase or a quantum string liquid. What is learned in this regard from the present study of a single string? A prerequisite for the existence of a quantum string liquid is that a single string is delocalized. If our conjecture that a single critical string is described by free-field theory turns out to be correct, this amounts to a considerable simplification. In Euclidean space time, the single free string world sheet is like a Gaussian membrane and a system of strings becomes a system of interacting Gaussian membranes, embedded in $2+1$ dimensions. This in turn is like a classical incommensurate system in three dimensions, which, although barely studied, appears as a tractable problem. For instance, it is known that the 3D incommensurate solid melts at a finite temperature in all cases. ${ }^{53}$ For the quantum case this means that the quantum-melting transition will occur at some finite value of the coupling constant, which in turn depends on the single-string quantum fluctuation as well as string-string interaction effects. Investigations addressing this many string problem are in progress, profiting from the simple fluctuation behavior of a single string.

\section{ACKNOWLEDGMENTS}

We thank S. A. Kivelson for stimulating discussions. The work of H.E. was supported by the Stichting voor Fundamenteel Onderzoek der Materie (FOM), which is financially supported by the Nederlandse Organisatie voor Wetenschappelijk Onderzoek (NWO). J.Z. acknowledges support by the Dutch Royal Academy of Sciences (KNAW).

\section{APPENDIX A: TRANSFER-MATRIX FORMALISM}

In this appendix we will discuss the specifics of the numerical calculations. This includes (a) a transfer-matrix formalism that is quite efficient numerically, (b) some specifics regarding the updates, and (c) some further discussion of the finite-temperature behavior of the directedness in the various $T=0$ phases.

The transfer matrix is constructed as follows. The partition function is

$$
\mathcal{Z}=\operatorname{Tr} e^{\mathcal{H}_{C l}+\mathcal{H}_{Q}}=\lim _{n \rightarrow \infty} \operatorname{Tr}\left(I T_{A} I T_{B}\right)^{n} .
$$

In the above formula $I$ is the identity operator, in our case a complete set of string configurations. We have chosen to split the $T$ matrix into a contribution from even and odd sites, or $A$ and $B$ sublattices (checkerboard decomposition),

$$
T_{A}=\exp \left[\frac{1}{n} \sum_{l=1}^{L / 2}\left(\mathcal{H}_{C l, 2 l}+\mathcal{H}_{Q, 2 l}\right)\right],
$$

with a similar expression for the odd sites. $\mathcal{H}(2 l)$ is the Hamiltonian of the even string element $2 l$, equal to Eq. (2.1) or (2.4) without the sum over string links. $L$ is the number of links in the chain. Because of the sublattice decomposition, $T_{A}$ is a simple product of local $T$ matrices and $\mathcal{Z}$ becomes

$$
\begin{aligned}
\mathcal{Z}= & \lim _{n \rightarrow \infty} \sum_{\left\{\mathbf{r}_{l, k}, \mathbf{r}_{l, k}^{\prime}\right\}} \prod_{k=1}^{n} \prod_{l=1}^{L / 2}\left\langle\left\{\mathbf{r}_{l}\right\}_{k}\left|t_{A}^{2 l, k}\right|\left\{\mathbf{r}_{l}\right\}_{k}^{\prime}\right\rangle \\
& \times\left\langle\left\{\mathbf{r}_{l}\right\}_{k}^{\prime}\left|t_{B}^{2 l+1, k}\right|\left\{\mathbf{r}_{l}\right\}_{k+1}\right\rangle .
\end{aligned}
$$

Each time slice is split in two subslices $\mathbf{r}$ and $\mathbf{r}^{\prime}$. The notation $\left\{\mathbf{r}_{l}\right\}_{k}$ denotes the set of positions $\mathbf{r}_{l}$ at the given time slice with index $k$. Note that the $t$ matrices are independent of $l$ and $k$ and these indices only label the position of the $t$ matrix in the 2D world sheet. The local $t$ matrices $t_{A}$ and $t_{B}$ depend only on three positions. For instance,

$$
\begin{aligned}
& \left\langle\left\{\mathbf{r}_{l}\right\}_{k}\left|t_{A}^{2 l, k}\right|\left\{\mathbf{r}_{l}\right\}_{k}^{\prime}\right\rangle \\
& \quad=\left\langle\mathbf{r}_{2 l-l, k} \mathbf{r}_{2 l, k} \mathbf{r}_{2 l+1, k}\left|t_{A}^{2 l, k}\right| \mathbf{r}_{2 l-1, k}^{\prime} \mathbf{r}_{2 l, k}^{\prime} \mathbf{r}_{2 l+1, k}^{\prime}\right\rangle,
\end{aligned}
$$

with the restriction $\mathbf{r}_{2 l-1, k}=\mathbf{r}_{2 l-1, k}^{\prime}$ and $\mathbf{r}_{2 l+1, k}=\mathbf{r}_{2 l+1, k}^{\prime}$. Since each link has eight different orientations, the local $t$ matrix connects in general $8 \times 8=64$ possibilities. However, most of the $t$ matrix elements are zero and it decomposes into subblocks, of which the biggest one is $3 \times 3$. The states that are connected via the local $t$ matrix, or the Hamiltonian, are listed in Fig. 16.

The local $t$ matrix at position $l$ and Trotter slice $k$ is defined as

$$
\left\langle\mathbf{r}_{l-1, k}, \mathbf{r}_{l, k}, \mathbf{r}_{l+1, k}\left|\exp \frac{1}{n} \mathcal{H}_{l}\right| \mathbf{r}_{l-1, k}^{\prime}, \mathbf{r}_{l, k}^{\prime}, \mathbf{r}_{l+1, k}^{\prime},\right\rangle
$$

The matrix elements depend on the positions of three members of the string $l-1, l$, and $l+1$. The positions of $l-1$ and $l+1$ are required to be identical $\mathbf{r}_{l-1, k}=\mathbf{r}_{l-1, k}^{\prime} \mathbf{r}_{l-1, k}^{\prime}$ in the two Trotter subslices involved, due to the checkerboard decomposition, but the position of member $l$ can be different, leading to off-diagonal matrix elements. The matrix elements 


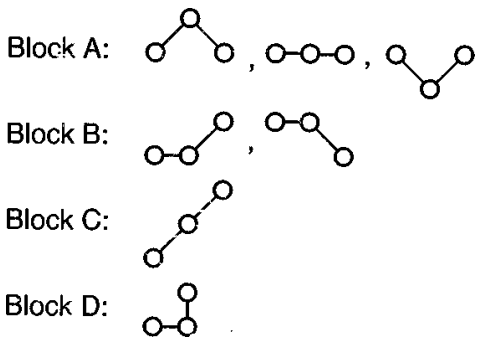

FIG. 16. Four subblocks of the local $t$ matrix. The other equivalent, symmetry related blocks are obtained by $\pi / 2$ rotations and reflections in the $x$ or $y$ axis.

of the $t$ matrix are easily found by first diagonalizing $\mathcal{H}_{\uparrow}$ and expanding the basis vectors in terms of the eigenvectors.

Block $A$ contains three configurations; see Fig. 16. We use the same order for the states as in the figure. Note that only half of the energy $\mathcal{K}$ of the diagonal link between $l$ -1 and $l$ and the link between $l$ and $l+1$ should be contributed to $l$. The Hamiltonian

$$
\mathcal{H}=\left(\begin{array}{ccc}
\mathcal{K} & \mathcal{T} & 0 \\
\mathcal{T} & 0 & \mathcal{T} \\
0 & \mathcal{T} & \mathcal{K}
\end{array}\right)
$$

is easily diagonalized. The eigenvalues are $\mathcal{K}, E_{+}$, and $E_{-}$. The $t$ matrix is

$$
\begin{gathered}
t=\left(\begin{array}{ccc}
t_{11} & t_{12} & t_{13} \\
t_{12} & t_{22} & t_{12} \\
t_{13} & t_{12} & T_{11}
\end{array}\right), \\
t_{11}=\frac{1}{2} e^{\mathcal{K} / n}+N_{+}^{2} e^{E_{+} / n}+N_{-}^{2} e^{E_{-} / n}, \\
t_{12}=N_{+}^{2} \alpha_{+} e^{E_{+} / n}+N_{-}^{2} \alpha_{-} e^{E_{-} / n}, \\
t_{13}=-\frac{1}{2} e^{\mathcal{K} / n}+N_{+}^{2} e^{E_{+} / n}+N_{-}^{2} e^{E_{-} / n}, \\
t_{22}=N_{+}^{2} \alpha_{+}^{2} e^{E_{+} / n}+N_{-}^{2} e^{E_{-} / n}, \\
\alpha_{ \pm}=\frac{E_{ \pm}-\mathcal{K}}{\mathcal{T}}, \quad N_{ \pm}=\frac{1}{\sqrt{2+\alpha_{ \pm}^{2}}} .
\end{gathered}
$$

Here $n$ is the number of Trotter slices and $\mathcal{K}, \mathcal{L}_{12}, \mathcal{L}_{22}, \mathcal{L}_{11}$, and $\mathcal{T}$ are the string model parameters.

Block $B$ contains two configurations, each with one horizontal and one diagonal link. Repeating the above procedure one finds

$$
\begin{gathered}
t=\left(\begin{array}{cc}
e^{D} \cosh (\mathcal{T} / n) & e^{D} \sinh (\mathcal{T} / n) \\
e^{D} \sinh (\mathcal{T} / n) & e^{D} \cosh (\mathcal{T} / n)
\end{array}\right), \\
D=\frac{\mathcal{K}}{2 n}+\frac{\mathcal{L}_{12}}{n} .
\end{gathered}
$$
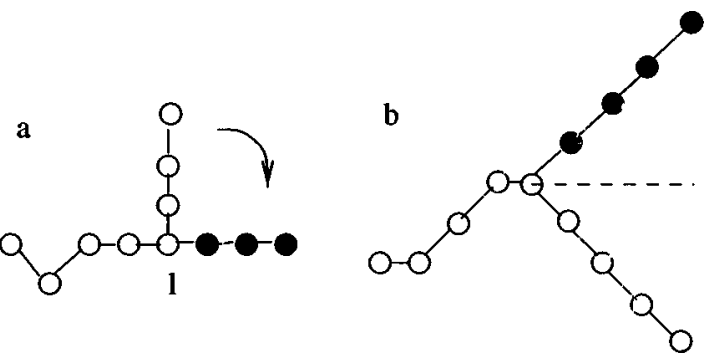

FIG. 17. Two additional Monte Carlo operations used for the simulations of the general string. (a) The $90^{\circ}$ rotation around position $l$ in this example turns a nondirected string into a directed one. (b) The mirror operation is important to quench defects in diagonal strings (mirror plane indicated by the dashed line).

Block $C$ contains a single configuration of two diagonal links, and the energy and $t$-matrix therefore contain $\mathcal{L}_{22}$,

$$
t=\exp \left(\frac{\mathcal{K}}{n}+\frac{\mathcal{L}_{22}}{n}\right)
$$

Block $D$ consists of a square corner between one horizontal and one vertical link and $\mathcal{L}_{11}$ is involved,

$$
t=\exp \left(\frac{\mathcal{L}_{11}}{n}\right)
$$

\section{APPENDIX B: GLOBAL MONTE CARLO MOVES}

For the Monte Carlo program to produce sensible results it is crucial to have operations that add and remove bends easily. We added global mirror and $\pi / 2$ rotation operations illustrated in Fig. 17.

In the latter case half of the string is rotated around any of the sites $l \in 2, \ldots, L-1$. This means that, for instance, the position of all holes $m>l$ is replaced by $\left(x_{m}, y_{m}\right) \rightarrow\left(x_{l}, y_{l}\right)$ $+\left[y_{m}-y_{l},-\left(x_{m}-x_{l}\right)\right]$. Such operations turn out to be very efficient: Completely wrapped high-temperature strings unwrap in just a couple of Monte Carlo steps at low temperature.

\section{APPENDIX C: DIRECTEDNESS AT LOW BUT FINITE TEMPERATURE}

Consider first the classical limit ( $\mathcal{T}=0$ and, for instance, the energy of the $\pi / 2$ corner $\mathcal{L}_{11}=1$ ). At zero temperature the string would be straight, running along (say) a $(1,0)$ direction. A local "corner" configuration of the type shown in Fig. 2(a) would be an excitation with energy $L_{11}$ (alternatively, one could consider two kinks). Clearly, a single corner suffices to destroy the directedness of the classical ground state. At any finite temperature, the probability of the occurrence of at least one corner is finite: $P$ $=N \exp \left(-\beta L_{11}\right)$. Hence directedness order cannot exist at finite temperatures, for the same reasons that long-range order is destroyed at any nonzero temperature in one dimension. In the simulations the string is of finite length and the infinite temperature limit of $N_{\mathrm{dir}}(T)$ is therefore not zero but rather a small but nonzero value ${ }^{54}(\sim 0.03$ for a domain wall of length 50). $N_{\mathrm{dir}}(T)$ is already close to this value for all temperatures of order $\mathcal{L}_{11}$ and larger. For an infinitely long 
domain wall $N_{\text {dir }}(T)$ drops very fast to zero with increasing temperature. At the other limit, for low $T$ where $T \ll \mathcal{L}_{11}$, $N_{\text {dir }}(T)$ increases very fast to 1 . Again, because the string is of finite length, it becomes directed already at a finite temperature: For all temperatures such that $\mathcal{L} \exp \left(-\beta \mathcal{L}_{11}\right)<1$ the string configurations in our simulations are typically completely directed. An infinitely long classical string becomes directed only at $T=0$, of course, since at any nonzero temperature always some corners will occur in a sufficiently long string.

For the quantum string, all the curves look strikingly similar to the classical one. When the temperature is very much higher than the kinetic term, $T \gg \mathcal{T}$, all curves merge together and the classical limit is reached. At low $T$, where $T \ll \mathcal{T}$, $N_{\text {dir }}(T)$ again increases very fast to 1 , as in the classical case; it reaches this value at a finite temperature for the finite length string. This is even true for the purely quantum string at the $X Y$ point, where all classical microscopic curvature energies are zero [see the dashed line in Fig. 4(a)]. We can understand this in terms of an effective corner or bend energy $\overline{\mathcal{L}}$ that is produced by the quantum fluctuations. As in the classical case the probability for the occurrence of a bend is proportional to $\sim \exp (-\beta \overline{\mathcal{L}})$. At zero temperature no bend is present and the string becomes directed. A finite length string effectively becomes directed already at a temperature such that $L \exp (-\beta \overline{\mathcal{L}})<1$. At intermediate temperatures, where the temperature is of the order of the kinetic term, things are more difficult and it is far from obvious what is going on. Especially in this region, all the various classical curvature energies may play a role and the interplay of these on the directedness is unclear. Nevertheless, as is clear from the data of Fig. 4(a), this region connects the high- and lowtemperature limits smoothly. Moreover, by comparing the results for the three quantum strings in this figure it is also clear that when the string is more quantum mechanical $N_{\text {dir }}(T)$ is higher.

We end this appendix with a brief qualitative description of our observations concerning spontaneous directedness at low but finite temperatures in regions of the phase diagram where the directed string has a type of ordering other than that already discussed. All the results apply to $\mathcal{L}_{11}=0$ and we refer to Table III in Sec. VII for a quick introduction to the various phases of the directed string problem and for the numbering $(\mathrm{I}-\mathrm{X})$ of the various phases.

The entire zero-temperature phase diagram of the directed string is reproduced.

Phase I is very stable with respect to bends. By "stable", we mean that finite strings do not change their appearance when increasing the temperature from zero to a moderately small temperature, of the order of $0.1 \mathcal{T}$.

Deep in the horizontal phase II (large positive $\mathcal{K}$ ) quantum fluctuations are strongly suppressed, and at the same time the string becomes susceptible to $\pi / 2$ corners. On the other hand, when we approach from phase II the boundaries with phases IV and V, the fluctuations increase and the string stiffens (Fig. 4). This is in agreement with the picture sketched before that quantum fluctuations orient the string.

Deep inside phase III the string changes constantly between horizontal zigzags and vertical zigzags. A $\pi / 2$ turn costs no extra energy. Again close to phase V quantum fluctuations have the effect of removing bends.

The Haldane phase $\mathrm{V}$ and the rough phase IV are very robust and a considerable fraction of $\pi / 2$ bends occurs only at relatively high temperatures of the order of $0.2 \mathcal{T}$.

In the slanted phase VII high temperatures are needed before down diagonal links come in. On the other hand, horizontal links are easily replaced by vertical ones. This only increases the energy very slightly, but the entropy gain is considerable. A typical low-temperature string is shown in Fig. 10 in Sec. VII. To zeroth order the horizontal and vertical links can be thought of as spinless fermions moving coherently along the string. In the dilute limit these links have only a weak interaction. The order of the links is conserved and at zero temperature the ground state has only horizontal links. However, our simulations indicate that for a small range of negative $L_{11}$ values a diagonal string with alternating horizontal and vertical links is favored. It is again the kinetic energy of the horizontal and vertical links that keeps the string oriented in the $(1,1)$ direction.
*Present address: KNMI, Postbus 201, 3730 AE De Bilt, The Netherlands.

${ }^{1}$ See, e.g., B. G. Levi, Phys. Today 49 (6), 17 (1996), and references therein.

${ }^{2}$ Y. Ando, G. S. Boebinger, A. Passner, T. Kimura, and K. Kishio, Phys. Rev. Lett. 75, 4662 (1995); Y. Ando, G. S. Boebinger, A. Passner, N. L. Wang, C. Geibel, and F. Steglich, ibid. 77, 2065 (1996); G. S. Boebinger, Y. Ando, A. Passner, T. Kimura, M. Okuya, J. Shimoyama, K. Kishio, K. Tamasaku, N. Ichikawa, and S. Uchida, ibid. 77, 5417 (1996).

${ }^{3}$ J. M. Tranquada, B. J. Sternlieb, J. D. Axe, Y. Nakamura, and S. Uchida, Nature (London) 375, 561 (1995); see also J. M. Tranquada, Physica B 241-243, 745 (1998).

${ }^{4}$ J. M. Tranquada, J. D. Axe, N. Ichikawa, A. R. Moodenbaugh, Y. Nakamura, and S. Uchida, Phys. Rev. Lett. 78, 338 (1997).

${ }^{5}$ K. Yamada, C. H. Lee, Y. Endoh, G. Shirane, R. J. Birgeneau, and M. A. Kastner, Physica C 282-287, 85 (1997).

${ }^{6} \mathrm{~K}$. Yamada et al. (unpublished).

${ }^{7}$ J. M. Tranquada, Physica C 282-287, 166 (1997).
${ }^{8}$ P. Dai, H. A. Mook, and F. Dogan, Phys. Rev. Lett. 80, 1738 (1998).

${ }^{9}$ J. Zaanen, M. L. Horbach, and W. van Saarloos, Phys. Rev. B 53, 8671 (1996).

${ }^{10}$ D. M. Ceperley, Rev. Mod. Phys. 67, 279 (1995).

${ }^{11}$ H. Eskes, R. Grimberg, W. van Saarloos, and J. Zaanen, Phys. Rev. B 54, 724 (1996).

${ }^{12}$ C. Nayak and F. Wilczek, Int. J. Mod. Phys. B 10, 2125 (1996); Phys. Rev. Lett. 78, 2465 (1997).

${ }^{13}$ See, e.g., G. Forgacs, R. Lipowsky, and Th. M. Nieuwenhuizen, in Phase Transitions and Critical Phenomena, edited by C. Domb and J. L. Lebowitz (Academic, New York, 1991), Vol. 14; Structure and Dynamics of Membranes, edited by R. Lipowsky and E. Sackmann (North-Holland, Amsterdam, 1995).

${ }^{14}$ J. Zaanen and O. Gunnarsson, Phys. Rev. B 40, 7391 (1989).

${ }^{15}$ D. Poilblanc and T. M. Rice, Phys. Rev. B 39, 9749 (1989); H. J. Schulz, Phys. Rev. Lett. 64, 1445 (1990); M. Kato, K. Machida, H. Nakanishi, and M. Fujita, J. Phys. Soc. Jpn. 59, 1047 (1990); M. Inui and P. B. Littlewood, Phys. Rev. B 44, 4415 (1991); J. 
A. Vergés, F. Guinea, and E. Louis, ibid. 46, 3562 (1992); T. Giamarchi and C. Lhuillier, ibid. 43, 12943 (1991); G. An and J. M. J. van Leeuwen, Phys. Rev. B 44, 9410 (1991); H. J. M. van Bemmel, D. F. B. ten Haaf, W. van Saarloos, J. M. J. van Leeuwen, and G. An, Phys. Rev. Lett. 72, 2442 (1994); J. Zaanen and P. B. Littlewood, Phys. Rev. B 50, 7222 (1994).

${ }^{16}$ U. Löw, V. J. Emery, K. Fabricius, and S. A. Kivelson, Phys. Rev. Lett. 72, 1918 (1994); V. J. Emery and S. Kivelson, Physica C 209, 597 (1993); S. A. Kivelson and V. J. Emery, Synth. Met. 80, 151 (1996).

${ }^{17}$ P. Prelovsek and X. Zotos, Phys. Rev. B 47, 5984 (1993); P. Prelovsek and I. Sega, ibid. 49, 15241 (1994).

${ }^{18}$ J. Zaanen and A. M. Oleś, Ann. Phys. (Leipzig) 5, 224 (1996).

${ }^{19}$ S. R. White and D. J. Scalapino, Phys. Rev. Lett. 80, 1272 (1998).

${ }^{20}$ V. J. Emery, S. A. Kivelson, and O. Zachar, Phys. Rev. B 56, 6120 (1997); A. H. Castro Neto, Z. Phys. B 102, 185 (1997); Phys. Rev. Lett. 78, 3931 (1997); A. H. Castro Neto, C. de C. Chamon, and C. Nayak, ibid. 79, 4629 (1997).

${ }^{21}$ J. Zaanen, O. Y. Osman, H. Eskes, and W. van Saarloos, J. Low Temp. Phys. 105, 569 (1996).

${ }^{22}$ Yu. A. Krotov, D.-H. Lee, and A. V. Balatsky, Phys. Rev. B 56, 8367 (1997).

${ }^{23}$ J. Zaanen, O. Y. Osman, H. Eskes, and W. van Saarloos (unpublished)

${ }^{24}$ See, e.g., R. Rajaraman, An Introduction to Solitons and Instantons in Quantum Field Theory (North-Holland, Amsterdam, 1989).

${ }^{25}$ M. den Nijs, in Phase Transitions and Critical Phenomena, edited by C. Domb and J. L. Lebowitz (Academic, London, 1988), Vol. 12.

${ }^{26}$ M. den Nijs and K. Rommelse, Phys. Rev. B 40, 4709 (1989).

${ }^{27}$ V. Sachan and D. J. Buttrey, Phys. Rev. B 51, 12742 (1995); K. Nakajima, Y. Endoh, S. Hosoya, J. Wada, D. Welz, H. M. Mayer, H. A. Graf, and M. Steiner, J. Phys. Soc. Jpn. 66, 809 (1997).

${ }^{28}$ S. R. White and D. J. Scalapino, cond-mat/9610104.

${ }^{29}$ H. E. Viertiö and T. M. Rice, J. Phys.: Condens. Matter 6, 7091 (1994).

${ }^{30}$ A. J. Heeger, S. Kivelson, J. R. Schrieffer, and W.-P. Su, Rev. Mod. Phys. 60, 781 (1988).

${ }^{31}$ S. A. Brazovski, Zh. Eksp. Teor. Fiz. 78, 677 (1980) [Sov. Phys. JETP 51, 342 (1980)].
${ }^{32}$ F. D. M. Haldane, Phys. Rev. Lett. 45, 1358 (1980).

${ }^{33}$ M. Suzuki, Prog. Theor. Phys. 56, 1454 (1976).

${ }^{34}$ J. D. Weeks, J. Chem. Phys. 67, 3106 (1977); J. D. Weeks, Phys. Rev. Lett. 52, 2160 (1984).

${ }^{35}$ J. D. Weeks, in Ordering in Strongly Fluctuating Condensed Matter Systems, edited by T. Riste (Plenum, New York, 1980), p. 293.

${ }^{36}$ H. van Beijeren and I. Nolten, in Structure and Dynamics of Surfaces II, edited by W. Schommers and P. von Blanckenhagen (Springer, Berlin, 1987).

${ }^{37}$ R. Botet, R. Jullien, and M. Kolb, Phys. Rev. B 28, 3914 (1983).

${ }^{38}$ U. Glaus and T. Schneider, Phys. Rev. B 30, 215 (1984).

${ }^{39}$ H. J. Schulz and T. Ziman, Phys. Rev. B 33, 6545 (1986).

${ }^{40}$ H. J. Schulz, Phys. Rev. B 34, 6372 (1986).

${ }^{41}$ These order parameters, as well as the string order parameter introduced below, are only nonzero once the height at an arbitrary point is specified. This can, e.g., be the guider point discussed in Sec. V or the reference height $h_{0}$ introduced in Ref. 26.

${ }^{42}$ F. D. M. Haldane, Phys. Rev. Lett. 50, 1153 (1983).

${ }^{43}$ I. Affleck, J. Phys.: Condens. Matter 1, 3047 (1989).

${ }^{44}$ I. Affleck, T. Kennedy, E. H. Lieb, and H. Tasaki, Phys. Rev. Lett. 59, 799 (1987); Commun. Math. Phys. 115, 477 (1988).

${ }^{45}$ U. Schollwöck and T. Jolicœur, Europhys. Lett. 30, 493 (1995).

${ }^{46}$ J. D. Johnson and B. M. McCoy, Phys. Rev. A 6, 1613 (1972).

${ }^{47}$ S. R. White, Phys. Rev. Lett. 69, 2863 (1992).

${ }^{48}$ P. Nightingale, J. Appl. Phys. 53, 7927 (1982).

${ }^{49}$ M. N. Barber, in Phase Transitions and Critical Phenomena, edited by C. Domb and J. L. Lebowitz (Academic, London, 1983), Vol. 8.

${ }^{50}$ K. Binder, Z. Phys. B 43, 119 (1981).

${ }^{51}$ S. N. Coppersmith, D. S. Fisher, B. I. Halperin, P. A. Lee, and W. F. Brinkman, Phys. Rev. B 25, 349 (1982).

${ }^{52}$ J. M. Tranquada, D. J. Buttrey, and V. Sachan, Phys. Rev. B 54, 12318 (1996); 55, R6113 (1997); P. Wochner, J. M. Tranquada, D. J. Buttrey, and V. Sachan, ibid. 57, 1066 (1998).

${ }^{53}$ M. E. Fisher and D. S. Fisher, Phys. Rev. B 25, 3192 (1982).

${ }^{54}$ In the high-temperature limit, the string becomes a self-avoiding walk on a two-dimensional lattice. For such a walk of length $N$, the radius of gyration $R_{g}$ grows as $R_{g} \sim N^{\nu}$, where $\nu=0.6$ in $D=2$. This implies that the directedness defined in this paper should go to zero as $N^{\nu-1}=N^{-0.4}$ for $N \rightarrow \infty$ in the hightemperature limit. 